\title{
Three nodal solutions of singularly perturbed elliptic equations on domains without topology
}

\section{Trois solutions nodales d'équations elliptiques avec perturbations singulières dans des domaines sans topologie}

\author{
Thomas Bartsch ${ }^{*, 1}$, Tobias Weth ${ }^{2}$ \\ Mathematisches Institut, Universität Giessen, Arndtstr. 2, 35392 Giessen, Germany
}

Received 16 June 2003; received in revised form 23 April 2004; accepted 15 July 2004

Available online 7 April 2005

\begin{abstract}
We prove the existence of three nodal solutions of the Dirichlet problem for the singularly perturbed equation $-\varepsilon \Delta u+u=$ $f(u)$ for $\varepsilon>0$ small on any bounded domain $\Omega \subset \mathbb{R}^{N}$. The nonlinearity $f$ grows superlinearly and subcritically. We do not require symmetry conditions nor conditions on the geometry or the topology of the domain. Two solutions have precisely two nodal domains, and the third solution has at most three nodal domains. A corresponding result holds true for the semilinear equation $-\Delta u+u=f(u)$ on $\Omega$ provided $\Omega$ contains a large ball.

(C) 2005 L'Association Publications de l'Institut Henri Poincaré. Published by Elsevier B.V. All rights reserved
\end{abstract}

\section{Résumé}

Nous prouvons l'existence de trois solutions nodales du problème de Dirichlet pour l'équation $-\varepsilon \Delta u+u=f(u)$ avec $\varepsilon>0$ petit dans tous les domaines bornés $\Omega \subset \mathbb{R}^{N}$. La nonlinéarité $f$ est sur-linéaire et sous-critique. Nous n'avons pas besoin de conditions de symétrie ni de conditions géométriques ou de conditions liées à la topologie du domaine. Deux solutions admettent exactement deux domaines nodeaux et la troisiems admet au plus trois domaines nodeaux. Un résultat correspondant est valide pour l'équation semi-linéaire $-\Delta u+u=f(u)$ dans le cas où $\Omega$ contient une grande boule.

(C) 2005 L'Association Publications de l'Institut Henri Poincaré. Published by Elsevier B.V. All rights reserved

\footnotetext{
* Corresponding author.

E-mail address: thomas.bartsch@math.uni-giessen.de (T. Bartsch).

1 Supported by DFG grant BA 1009/15-1

2 Supported by DFG grant WE 2821/2-1.
}

0294-1449/\$ - see front matter (C) 2005 L'Association Publications de l'Institut Henri Poincaré. Published by Elsevier B.V. All rights reserved doi:10.1016/j.anihpc.2004.07.005 


\section{Introduction}

In this paper we are concerned with the singularly perturbed problem

$$
\begin{cases}-\varepsilon \Delta u+a u=f(u), & x \in \Omega, \varepsilon \searrow 0 ; \\ u=0 & \text { on } \partial \Omega\end{cases}
$$

and with the semilinear Dirichlet problem

$$
\begin{cases}-\Delta u+a u=f(u), & x \in \Omega \\ u=0 & \text { on } \partial \Omega .\end{cases}
$$

Here $\Omega \subset \mathbb{R}^{N}, N \geqslant 2$, is a bounded domain, and $a>0$ is fixed. The nonlinearity $f \in \mathcal{C}^{1}(\mathbb{R})$ grows superlinearly and subcritically. A model nonlinearity is

$$
f(u)=\sum_{i=1}^{k} c_{i}|u|^{p_{i}-2} u^{+}+\sum_{j=1}^{l} d_{j}|u|^{q_{j}-2} u^{-},
$$

where $p_{i}, q_{j} \in\left(2,2^{*}\right), c_{i}, d_{j}>0$ for $1 \leqslant i \leqslant k, 1 \leqslant j \leqslant l$ and $u^{+}=\max \{u, 0\}, u^{-}=\min \{u, 0\}$. Here $2^{*}$ denotes the critical Sobolev exponent, that is, $2^{*}:=\frac{2 N}{N-2}$ for $N \geqslant 3$, and $2^{*}=\infty$ for $N=2$.

In the situation that we consider, it is well known that (1.1) and (1.2) have three solutions (cf. [26]), one positive solution $u_{+}$, one negative solution $u_{-}$, and one sign changing solution $u_{1}$ (cf. [5,10,11]). No conditions on $\Omega$ or $\varepsilon$ are required for this. Morse theory and degree theory do not yield any further solutions. In fact, generically the onesign solutions are of mountain pass type with Morse index 1, and the nodal solution has Morse index 2. Together with the trivial solution 0 which has Morse index 0 the Morse inequalities are satisfied. The situation changes when $f$ is odd, or when the domain is radially symmetric or has nontrivial topology. If $f$ is odd then there are infinitely many solutions of (1.2) (cf. [1]) which change sign (cf. [2]). Related multiplicity results can be found in $[18,19]$. If $\Omega$ is radially symmetric then there exist radially symmetric solutions with prescribed number of nodal domains (cf. [24]). If $\Omega$ has nontrivial topology or geometry then the existence of multiple positive solutions of (1.1) for small $\varepsilon>0$ has been obtained in $[8,9,20]$, for instance.

In this paper we prove the existence of three nodal solutions $u_{1}, u_{2}, u_{3}$ of (1.2) provided $\Omega$ contains a large ball $B_{R}(0)$. As a consequence we obtain three nodal solutions of (1.1) for small $\varepsilon>0$, without any conditions on $\Omega$ except being bounded. For both results $\Omega$ may be contractible or even convex. Of course, we do not require that $f$ is odd (nor homogeneous). The solutions $u_{1}$ and $u_{2}$ have precisely two nodal domains. In fact, one can prove that as $R \rightarrow \infty$ the positive part $u_{1}^{+}$of $u_{1}$ converges (after translations) to a positive ground state of $-\Delta u+a u=f(u)$ on $\mathbb{R}^{N}$, and the negative part $u_{1}^{-}$of $u_{1}$ converges to a negative ground state. The same holds for $u_{2}$. Clearly the above mentioned results on positive (and similarly, negative) solutions can be combined with our theorems in order to obtain new multiplicity results. Our proof is based on a new existence mechanism for nodal solutions of (1.2) (and (1.1)) using a variational approach. A surprising feature of this mechanism is that, although the second solution $u_{2}$ has precisely two nodal domains it has Morse index $N+1$ (if nondegenerate). Similarly, the third solution $u_{3}$ has at most three nodal domains and Morse index $N+2$ (if nondegenerate). The existence of such solutions has not been observed so far.

In order to state our results we first formulate our assumptions:

(f $\left.\mathrm{f}_{1}\right) f \in \mathcal{C}^{1}(\mathbb{R}), f(0)=f^{\prime}(0)=0$.

$\left(\mathrm{f}_{2}\right)$ There exists $p \in\left(2,2^{*}\right)$ such that

$$
\left|f^{\prime}(t)\right| \leqslant C\left(1+|t|^{p-2}\right) \quad \text { for all } t \in \mathbb{R} .
$$

$\left(f_{3}\right) f^{\prime}(t)>f(t) / t$ for all $t \neq 0$.

$\left(\mathrm{f}_{4}\right)$ There exist $\theta>2$ such that $0<\theta F(t) \leqslant t f(t)$ for all $t \in \mathbb{R}$. 
Here $F(t):=\int_{0}^{t} f(s) \mathrm{d} s$ is a primitive of $f$. These assumptions in particular hold for the model nonlinearity (1.3). Our main results are the following:

Theorem 1.1. Suppose that $\left(\mathrm{f}_{1}\right)-\left(\mathrm{f}_{4}\right)$ are satisfied. Then, for any bounded domain $\Omega$, there is $\varepsilon_{0}>0$ such that for $\varepsilon \in\left(0, \varepsilon_{0}\right)$ the problem (1.1) has at least three nodal solutions $v_{1}, v_{2}, v_{3}$. Moreover, $v_{1}$ and $v_{2}$ have precisely two nodal domains, and $v_{3}$ has at most three nodal domains.

Theorem 1.2. Suppose that $\left(\mathrm{f}_{1}\right)-\left(\mathrm{f}_{4}\right)$ are satisfied. Then there is $R>0$ such that for any bounded domain $\Omega \subset \mathbb{R}^{N}$ with $B_{R}(0) \subset \Omega$, problem (1.2) has at least three nodal solutions $u_{1}, u_{2}, u_{3}$. Moreover, $u_{1}$ and $u_{2}$ have precisely two nodal domains, and $u_{3}$ has at most three nodal domains.

Using the scaling $v_{i}(x)=u_{i}((x-Q) / \sqrt{\varepsilon})$, some $Q \in \Omega$, one easily sees that Theorem 1.1 follows from Theorem 1.2. A strengthened version of Theorem 1.1 is equivalent to Theorem 1.2. In fact, one simply includes in Theorem 1.1 that $\varepsilon_{0}=\varepsilon_{0}(\Omega)$ only depends on the largest $r>0$ such that $B_{r}(Q)=\left\{x \in \mathbb{R}^{N}|| x-Q \mid<r\right\} \subset \Omega$ for some $Q \in \Omega$. Observe that we do not assume that the ground state solutions of (1.2) on $\mathbb{R}^{N}$ are unique modulo translations as is usually required when considering singularly perturbed problems.

Let us make a few remarks concerning the proof of Theorem 1.2. We use the energy functional

$$
\Phi: H^{1}\left(\mathbb{R}^{N}\right) \rightarrow \mathbb{R}, \quad \Phi(u)=\frac{1}{2} \int\left(|\nabla u|^{2}+a u^{2}\right) \mathrm{d} x-\int F(u(x)) \mathrm{d} x,
$$

and its restriction $\Psi=\left.\Phi\right|_{H_{0}^{1}(\Omega)}$. The one-sign solutions $u_{+}, u_{-}$mentioned above are obtained from the mountain pass theorem applied to $\Psi$. Let $c_{ \pm}=\min \left\{\Phi(u) \mid \pm u>0, \Phi^{\prime}(u)=0\right\}$ be the least energy of a positive, respectively negative, critical point of $\Phi$. All nodal solutions of (1.2) lie in the set

$$
\mathcal{M}_{\Omega}=\left\{u \in H_{0}^{1}(\Omega) \mid u^{+}, u^{-} \neq 0, \Psi^{\prime}(u) u^{+}=0=\Psi^{\prime}(u) u^{-}\right\} .
$$

We prove that $c_{1}=\left.\inf \Psi\right|_{\mathcal{M}_{\Omega}} \geqslant c_{+}+c_{-}$is achieved and that every $u \in \mathcal{M}_{\Omega}$ with $\Psi(u)=c_{1}$ is a critical point of $\Psi$, hence a nodal solution of (1.2). This last statement is not as obvious as it may seem. The set $\mathcal{M}_{\Omega}$ is not a manifold, one cannot talk about vector fields on $\mathcal{M}_{\Omega}$ and one cannot easily construct deformations on $\mathcal{M}_{\Omega}$. Consequently, min-max values for $\Psi$ on $\mathcal{M}_{\Omega}$ are not automatically critical points of $\Psi$. We mention this point because it has been overlooked in too many papers. We refer the reader to [6] for a proof of the fact that $\mathcal{M}_{\Omega} \cap H^{2}(\Omega)$ is a codimension 2 submanifold of $H^{2}(\Omega)$.

We also prove that every nodal solution of $(1.2)$ with $\Psi(u)<c_{1}+\min \left\{c_{+}, c_{-}\right\}$has precisely two nodal domains. The solution $u_{1}$ in Theorem 1.2 is obtained as a minimizer of $\Psi$ on $\mathcal{M}_{\Omega}$. In order to obtain the new solutions $u_{2}, u_{3}$ we consider the topology of the pair $\left(\Psi^{v} \cup \mathcal{E}, \mathcal{E}\right)$ for $v \in \mathbb{R}$ where $\Psi^{v}$ is a sublevel set as usual, and $\mathcal{E}$ is a thickened version of $\Psi^{c_{+}+c_{-}} \cup P \cup(-P)$. Here $P=\left\{u \in H_{0}^{1}(\Omega) \mid u \geqslant 0\right.$ a.e. $\}$ is the cone of nonnegative functions in $H_{0}^{1}(\Omega)$. We prove that $H_{N+1}\left(\Psi^{v} \cup \mathcal{E}, \mathcal{E}\right)$ is trivial if $v>c_{1}$ is close to $c_{1}$. We also produce a nontrivial element $\xi \in H_{N+1}\left(\Psi^{\nu_{0}} \cup \mathcal{E}, \mathcal{E}\right)$ for some $\nu_{0} \in\left(c_{1}, c_{1}+\min \left\{c_{+}, c_{-}\right\}\right)$. This yields a critical point $u_{2}$ of $\Psi$ in $\Psi^{\nu} \backslash \mathcal{E}$ with $\Psi\left(u_{2}\right)>\Psi\left(u_{1}\right)=c_{1}$. Since $u_{2} \notin \mathcal{E}$ it must change sign, hence by our energy estimate it has precisely two nodal domains. Moreover, its $(N+1)$-th critical group is nontrivial which implies that in the nondegenerate case $u_{2}$ has Morse index $N+1$. Finally we prove that $\xi$ maps to 0 in $H_{N+1}\left(\Psi^{v} \cup \mathcal{E}, \mathcal{E}\right)$ for $v$ large. This yields the third solution $u_{3} \notin \mathcal{E}$. If isolated this solution has a nontrivial critical group in dimension $N+2$, which implies that in the nondegenerate case $u_{3}$ has Morse index $N+2$. Again an energy estimate yields that $u_{3}$ has at most three nodal domains.

The decisive step is the construction of $\xi \neq 0 \in H_{N+1}\left(\Psi^{\nu_{0}} \cup \mathcal{E}, \mathcal{E}\right)$. Here a new generalized barycenter map $\beta: L^{2}\left(\mathbb{R}^{N}\right) \backslash\{0\} \rightarrow \mathbb{R}^{N}$ plays an important role. This map is continuous and equivariant with respect to the standard actions of the group of Euclidean motions on $\mathbb{R}^{N}$ and on $L^{2}\left(\mathbb{R}^{N}\right)$. This means that for $u \in L^{2}\left(\mathbb{R}^{N}\right) \backslash\{0\}, b \in \mathbb{R}^{N}$, $A \in O(N)$, the barycenter of $v(x):=u(A x+b)$ is $\beta(v)=A^{-1}(\beta(u)-b)$. We give an explicit construction of such a map independent of any choices of cut-off functions and the like. This provides barycenter maps on $L^{2}\left(\mathbb{R}^{N}\right)$ and 
subspaces thereof like $H_{0}^{1}(\Omega)$ whose construction does not depend on $\Omega$. We believe that $\beta$ will have further useful applications, in particular when variations or scalings of the domain are involved. It can be used, for instance, to obtain a simpler approach to some existence and multiplicity results on superlinear elliptic equations on domains with topology, cf. [8,7] and [12, Chapter 6].

The paper is organized as follows. The generalized barycenter map is constructed in Section 2. In fact, we construct a generalized barycenter map $\beta_{p}: L^{p}\left(\mathbb{R}^{N}\right) \backslash\{0\} \rightarrow \mathbb{R}^{N}$ for any $p \in[1, \infty)$. This construction is completely independent from any nonlinear equation and of independent interest. In Section 3 we provide various estimates for the functional $\Phi$ on $H^{1}\left(\mathbb{R}^{N}\right)$ which are essential for the construction of the nontrivial element in the homology of $\left(\Psi^{\nu_{0}} \cup \mathcal{E}, \mathcal{E}\right)$. The set $\mathcal{M}_{\Omega}$ is studied in some detail in Section 4. Finally, Theorem 1.2 is proved in Section 5 .

By $|\cdot|_{p}$ we denote the usual norm of $L^{p}\left(\mathbb{R}^{n}\right)$ for $1 \leqslant p \leqslant \infty$. For a subset $A$ of a topological space we denote by $\operatorname{int}(A)$ the interior of $A$.

\section{A generalized barycenter map on $L^{p}\left(\mathbb{R}^{N}\right)$}

For $b \in \mathbb{R}^{N}$ let $\tau_{b}: \mathbb{R}^{N} \rightarrow \mathbb{R}^{N}$ be the translation $\tau_{b}(x):=x+b$. The group of Euclidean motions on $\mathbb{R}^{N}$ is given by $\mathcal{G}:=\left\{\tau_{b} \circ A \mid b \in \mathbb{R}^{N}, A \in O(N)\right\}$. It acts on $\mathbb{R}^{N}$ (from the left) in the usual way and induces a (left) action on the space of maps $\mathbb{R}^{N} \rightarrow M, M$ any set, as follows: $g * u:=u \circ g^{-1}$ for $g \in \mathcal{G}, u: \mathbb{R}^{N} \rightarrow M$. Explicitly this means for $g=\tau_{b} \circ A$ :

$$
g * u(x)=u\left(A^{-1}(x-b)\right) .
$$

We call a map $\beta: L^{p}\left(R^{N}\right) \backslash\{0\} \rightarrow \mathbb{R}^{N}$ a generalized barycenter map on $L^{p}\left(\mathbb{R}^{N}\right)$ if it is continuous and $\mathcal{G}$-equivariant, that is, $\beta(g * u)=g(\beta(u))$ for $g \in \mathcal{G}, u \in L^{p}\left(\mathbb{R}^{N}\right) \backslash\{0\}$. For $b \in \mathbb{R}^{N}, A \in O(N)$ and $u \in$ $L^{p}\left(\mathbb{R}^{N}\right) \backslash\{0\}$ this implies that

$$
\beta(u(\cdot-b))=\beta(u)+b, \quad \text { and } \quad \beta\left(u \circ A^{-1}\right)=A(\beta(u)) .
$$

As a consequence, if $u \circ A=u$ then $\beta(u)=A(\beta(u))$. Thus an even function, especially a radial function $u \in$ $L^{p}\left(\mathbb{R}^{N}\right) \backslash\{0\}$ has barycenter $\beta(u)=0$. More generally, if $u \in L^{p}\left(\mathbb{R}^{N}\right) \backslash\{0\}$ is invariant with respect to a subgroup $G \subset O(N)$ then $\beta(u) \in\left(\mathbb{R}^{N}\right)^{G}=\left\{x \in \mathbb{R}^{N} \mid g x=x\right.$ for all $\left.g \in G\right\}$.

The main result of this section is as follows:

Theorem 2.1. For any $p \in[1, \infty)$ there exists a generalized barycenter map $\beta=\beta_{p}: L^{p}\left(\mathbb{R}^{N}\right) \backslash\{0\} \rightarrow \mathbb{R}^{N}$ which satisfies $\beta(|u|)=\beta(u)$.

Proof. We fix $p \in[1, \infty)$ and write $L^{p}=L^{p}\left(\mathbb{R}^{N}\right)$. By $\mathcal{B}$ we denote the Banach space of all continuous functions $f: \mathbb{R}^{N} \rightarrow \mathbb{R}$ with $f(x) \rightarrow 0$ as $|x| \rightarrow \infty$, endowed with the supremum norm

$$
|f|_{\infty}=\max _{x \in \mathbb{R}^{N}}|f(x)| \text {. }
$$

It is easy to see that for $u \in L^{p}$ the function

$$
\hat{u}: \mathbb{R}^{N} \rightarrow[0, \infty), \quad \hat{u}(x)=\int_{B_{1}(x)}|u(y)|^{p} \mathrm{~d} y,
$$

is an element of $\mathcal{B}$. Moreover, for $u \in L^{p}$ we have

$$
\hat{u}=0 \quad \text { if and only if } u=0 .
$$

We also note that for $u, v \in L^{p}$ and $x \in \mathbb{R}^{N}$ 


$$
\begin{aligned}
|\hat{u}(x)-\hat{v}(x)| & \leqslant\left.\int_{B_{1}(x)}|| u\right|^{p}-|v|^{p}\left|\leqslant p \int_{B_{1}(x)}\right| u-v \mid(|u|+|v|)^{p-1} \\
& \leqslant p\left(\int_{B_{1}(x)}|u-v|^{p}\right)^{1 / p} \cdot\left(\int_{B_{1}(x)}(|u|+|v|)^{p}\right)^{(p-1) / p} \\
& \leqslant p 2^{p-1}|u-v|_{p} \cdot\left(|u|_{p}^{p}+|v|_{p}^{p}\right)^{(p-1) / p}
\end{aligned}
$$

hence

$$
|\hat{u}-\hat{v}|_{\infty} \leqslant p 2^{p-1}|u-v|_{p} \cdot\left(|u|_{p}^{p}+|v|_{p}^{p}\right)^{(p-1) / p} .
$$

Consequently the function $L^{p} \rightarrow \mathcal{B}, u \mapsto \hat{u}$ is continuous. For $u \in L^{p}$ we consider the set

$$
\Omega(u)=\left\{x \in \mathbb{R}^{N}: \hat{u}(x) \geqslant \frac{|\hat{u}|_{\infty}}{2}\right\} .
$$

If $u \neq 0$ then $\Omega(u) \subset \mathbb{R}^{N}$ is compact and has nonempty interior. Moreover,

$$
\beta_{1}(u):=\int_{\Omega(u)}\left(\hat{u}(x)-\frac{|\hat{u}|_{\infty}}{2}\right) \mathrm{d} x>0 \quad \text { for every } u \in L^{p} \backslash\{0\} .
$$

Therefore setting

$$
\beta_{0}(u):=\int_{\Omega(u)} x\left(\hat{u}(x)-\frac{|\hat{u}|_{\infty}}{2}\right) \mathrm{d} x \in \mathbb{R}^{N},
$$

the function

$$
\beta: L^{p} \backslash\{0\} \rightarrow \mathbb{R}^{N}, \quad \beta(u)=\frac{\beta_{0}(u)}{\beta_{1}(u)}
$$

is well defined. One easily checks $\beta(g * u)=g(\beta(u))$ for an Euclidean motion $g \in \mathcal{G}, u \in L^{p} \backslash\{0\}$, so $\beta$ is $\mathcal{G}$-equivariant. Since we also have $\beta(|u|)=\beta(u)$, it remains to prove that the maps $\beta_{1}$ and $\beta_{0}$ are continuous. We only consider $\beta_{0}$, the argument for $\beta_{1}$ is similar. Consider a sequence $u_{n} \rightarrow u$ in $L^{p} \backslash\{0\}$. Then $\hat{u}_{n} \rightarrow \hat{u}$ in $\mathcal{B}$, so for $n$ large enough we have

$$
\frac{1}{2} \leqslant \frac{\hat{u}_{n}(x)}{\left|\hat{u}_{n}\right|_{\infty}} \leqslant \frac{\hat{u}(x)}{|\hat{u}|_{\infty}}+\frac{1}{4} \quad \text { if } \hat{u}_{n}(x) \geqslant \frac{\left|\hat{u}_{n}\right|_{\infty}}{2} .
$$

Setting

$$
K:=\left\{x \in \mathbb{R}^{N}: \hat{u}(x) \geqslant|\hat{u}|_{\infty} / 4\right\}
$$

we see that $K$ is compact and that $\Omega\left(u_{n}\right) \subset K$ for $n$ large. Therefore

$$
\beta_{1}\left(u_{n}\right)=\int_{K}\left(\hat{u}_{n}(x)-\frac{\left|\hat{u}_{n}\right|_{\infty}}{2}\right)^{+} \mathrm{d} x \rightarrow \int_{K}\left(\hat{u}(x)-\frac{|\hat{u}|_{\infty}}{2}\right)^{+} \mathrm{d} x=\beta_{1}(u)
$$

by Lebesgue's dominated convergence theorem because the integrand in $\beta_{1}\left(u_{n}\right)$ converges pointwise as $n \rightarrow \infty$, and it is bounded above uniformly by $\max \left\{\left|\hat{u}_{n}\right|_{\infty} / 2: n \in \mathbb{N}\right\}$. 


\section{The functional on $\mathbb{R}^{N}$}

For matters of convenience we assume $a=1$ in (1.2) from now on, the general case follows by obvious modifications. We set $E=H^{1}\left(\mathbb{R}^{N}\right)$ endowed with the standard scalar product

$$
(u, v)=\int_{\mathbb{R}^{N}}(\nabla u \nabla v+u v) \mathrm{d} x, \quad u, v \in E,
$$

and we denote the induced norm by $\|\cdot\|$. It is well known that, as a consequence of $\left(\mathrm{f}_{1}\right)$ and $\left(\mathrm{f}_{2}\right)$, the functional

$$
\Phi: E \rightarrow \mathbb{R}, \quad \Phi(u)=\frac{1}{2}\|u\|^{2}-\int F(u(x)) \mathrm{d} x
$$

is of class $C^{2}$, and that critical points of $\Phi$ are weak solutions of (1.2) with $a=1$ on $\Omega=\mathbb{R}^{N}$.

Let us fix some notation. We define $\Phi^{v}=\{u \in E: \Phi(u) \leqslant v\}$ as usual and $A^{v}=A \cap \Phi^{v}$ for $A \subset E, v \in \mathbb{R}$. Moreover, for $u \in E$ and $A \subset E$ we put

$$
\operatorname{dist}_{1}(u, A)=\inf _{v \in A}\|u-v\| \quad \text { and } \quad \operatorname{dist}_{2}(u, A)=\inf _{v \in A}|u-v|_{2} .
$$

We also define for $\varepsilon>0$ the closed neighborhoods

$$
U_{\varepsilon}^{1}(A)=\left\{u \in E: \operatorname{dist}_{1}(u, A) \leqslant \varepsilon\right\}
$$

and

$$
U_{\varepsilon}^{2}(A)=\left\{u \in E: \operatorname{dist}_{2}(u, A) \leqslant \varepsilon\right\}
$$

of $A$. Every nontrivial critical point of $\Phi$ is contained in the Nehari manifold

$$
\mathcal{N}=\left\{u \in E \backslash\{0\} \mid \Phi^{\prime}(u) u=0\right\} .
$$

We set

$$
c_{ \pm}=\inf \{\Phi(u) \mid u \in \mathcal{N}, \pm u \geqslant 0\}
$$

and

$$
d_{ \pm}=\inf \{\|u\| \mid u \in \mathcal{N}, \pm u \geqslant 0\}
$$

and consider the sets

$$
\mathcal{K}^{ \pm}=\left\{u \in E \mid \pm u>0, \Phi(u)=c_{ \pm}, \Phi^{\prime}(u)=0\right\}
$$

and

$$
\mathcal{K}_{\text {rad }}^{ \pm}=\left\{u \in \mathcal{K}^{ \pm} \mid u \text { is radially symmetric }\right\} .
$$

Using the compactness of the embeddings (cf. [21])

$$
E_{\text {rad }}:=\{u \in E: u \text { radially symmetric }\} \hookrightarrow L^{s}, \quad s \in\left(2,2^{*}\right)
$$

one can show that $\mathcal{K}_{\text {rad }}^{ \pm}$is compact and nonempty. Moreover, every one-sign solution of (1.2) on $\Omega=\mathbb{R}^{N}$ is radially symmetric around some point in $\mathbb{R}^{N}$ (see [17]) and therefore

$$
\mathcal{K}^{ \pm}=\mathbb{R}^{N} * \mathcal{K}_{\text {rad }}^{ \pm}=\left\{y * u: y \in \mathbb{R}^{N}, u \in \mathcal{K}_{\text {rad }}^{ \pm}\right\}
$$

Here and in the sequel we write $y * u(x):=u(x-y)$ for $y \in \mathbb{R}^{N}$ and $u \in E$. We also note that $\left(\mathrm{f}_{4}\right)$ implies

$$
\Phi(u) \geqslant\left(\frac{1}{2}-\frac{1}{\theta}\right)\|u\|^{2} \quad \text { for every } u \in \mathcal{N} .
$$

Moreover we will use the following properties of $\Phi$ which are well known consequences of $\left(\mathrm{f}_{1}\right)-\left(\mathrm{f}_{4}\right)$; $\mathrm{cf}$. [8, Lemma 2.1, Lemma 2.2]. 
Lemma 3.1. $\mathcal{N}$ is a $C^{1}$-submanifold of $E$ which is diffeomorphic to the unit sphere in $E$ by radial projection. Moreover,

(i) $\Phi^{\prime \prime}(u)(u, u)<0$ for every $u \in \mathcal{N}$;

(ii) for every $u \in \mathcal{N}$ the function $t \mapsto \Phi(t u)$ is strictly increasing on $(0,1)$, strictly decreasing on $(1, \infty)$, and $\lim _{t \rightarrow \infty} \Phi(t u)=-\infty$

(iii) $c_{ \pm}>0$;

(iv) $d_{ \pm}>0$

(v) $\inf \Phi(\mathcal{N})=\min \left\{c_{+}, c_{-}\right\}$;

(vi) if $u \in \mathcal{N}$ satisfies $u \geqslant 0$ and $\Phi(u)=c_{+}$, then $u \in \mathcal{K}^{+}$;

(vii) if $u \in \mathcal{N}$ satisfies $u \leqslant 0$ and $\Phi(u)=c_{-}$, then $u \in \mathcal{K}^{-}$.

Lemma 3.2. Let $\left(u_{n}\right) \in \mathcal{N}$ be a sequence such that $\pm u_{n} \geqslant 0$ for all $n$ and $\Phi\left(u_{n}\right) \rightarrow c_{ \pm}$. Then $\operatorname{dist}_{1}\left(u_{n}, \mathcal{K}^{ \pm}\right) \rightarrow 0$.

Proof. We only consider the case where $u_{n} \geqslant 0$ for all $n \in \mathbb{N}$. It is sufficient to show that $\operatorname{dist}_{1}\left(u_{n}, \mathcal{K}^{+}\right) \rightarrow 0$ holds for a subsequence. We define

$$
\begin{aligned}
& f_{+}: \mathbb{R} \rightarrow \mathbb{R}, \quad f_{+}(t)= \begin{cases}f(t), & t \geqslant 0, \\
0, & t<0,\end{cases} \\
& F_{+}(t):=\int_{0}^{t} f_{+}(s) \mathrm{d} s \quad \text { for } t \in \mathbb{R},
\end{aligned}
$$

and

$$
\begin{aligned}
& \Phi_{+} \in C^{2}(E), \quad \Phi_{+}(u)=\frac{1}{2}\|u\|^{2}-\int F_{+}(u(x)) \mathrm{d} x, \\
& \mathcal{N}_{+}=\left\{u \in E \backslash\{0\} \mid \Phi_{+}^{\prime}(u) u=0\right\} .
\end{aligned}
$$

We clearly have

$$
\Phi_{+}(u)=\Phi(u), \quad \text { and } \quad \Phi_{+}^{\prime}(u)=\Phi^{\prime}(u) \quad \text { for every } u \in E, u \geqslant 0
$$

and $c_{+}=\inf _{u \in \mathcal{N}_{+}} \Phi_{+}(u)$. In particular we infer that

$$
u_{n} \in \mathcal{N}_{+}, \quad \Phi_{+}\left(u_{n}\right)=\Phi\left(u_{n}\right)
$$

for every $n$, and that $\left(u_{n}\right)$ is a minimizing sequence for $\Phi_{+}$on $\mathcal{N}_{+}$. Hence $\left\|u_{n}\right\|$ is bounded by (3.2). Moreover, $\mathcal{N}_{+} \subset E$ is a $C^{1}$-submanifold of codimension one, as follows essentially by the proof of [8, Lemma 2.2]. Now Ekeland's variational principle (see e.g. [25]) yields a sequence $\left(v_{n}\right) \subset \mathcal{N}_{+}$with

$$
\begin{aligned}
& \Phi_{+}\left(v_{n}\right) \rightarrow c_{+}, \\
& \left(\Phi_{+} \mid \mathcal{N}_{+}\right)^{\prime}\left(v_{n}\right) \rightarrow 0, \\
& \left\|u_{n}-v_{n}\right\| \rightarrow 0 .
\end{aligned}
$$

We claim that

$$
\sup _{y \in \mathbb{R}^{N}} \int_{B_{1}(0)}\left(y * v_{n}\right)^{2}(x) \mathrm{d} x \not \rightarrow 0 \quad \text { as } n \rightarrow \infty .
$$

Indeed, if on the contrary $\sup _{y \in \mathbb{R}^{N}} \int_{B_{1}(0)}\left(y * v_{n}\right)^{2}(x) \mathrm{d} x \rightarrow 0$, then $\left|v_{n}\right|_{s} \rightarrow 0$ for $s \in\left(2,2^{*}\right)$ by [22, Lemma I.1]. However, as a consequence of $\left(\mathrm{f}_{1}\right)$ and $\left(\mathrm{f}_{3}\right)$ there exists a constant $C_{0}>0$ such that 


$$
|f(t)| \leqslant \frac{1}{2}|t|+C_{0}|t|^{p-1} \quad \text { for all } t \in \mathbb{R}
$$

Since $v_{n} \in \mathcal{N}_{+}$, we have

$$
\left\|v_{n}\right\|^{2}=\int_{\mathbb{R}^{N}} f_{+}\left(v_{n}\right) v_{n} \mathrm{~d} x \leqslant \frac{1}{2}\left|v_{n}^{+}\right|_{2}^{2}+C_{0}\left|v_{n}^{+}\right|_{p}^{p} \leqslant \frac{1}{2}\left|v_{n}\right|_{2}^{2}+C_{0}\left|v_{n}\right|_{p}^{p} \leqslant \frac{1}{2}\left\|v_{n}\right\|^{2}+o(1),
$$

which implies $\left\|v_{n}\right\| \rightarrow 0$, contrary to (3.4) and the fact that $\left\|u_{n}\right\| \geqslant d_{+}>0$ for all $n$. This shows (3.5), and hence there are $y_{n} \in \mathbb{R}^{N}, n \in \mathbb{N}$ such that for a subsequence - still denoted by $\left(v_{n}\right)$ - we have

$$
w_{n}:=y_{n} * v_{n} \rightarrow w \in E \backslash\{0\} .
$$

We note that $w \geqslant 0$, since $u_{n} \geqslant 0$ and $y_{n} * u_{n} \rightarrow w$. Now we consider $J \in C^{1}(\mathbb{R})$ given by $J(u):=\Phi_{+}^{\prime}(u) u$. By (3.3),

$$
o(1)=\left(\left.\Phi_{+}\right|_{\mathcal{N}_{+}}\right)^{\prime}\left(w_{n}\right)=\Phi_{+}^{\prime}\left(w_{n}\right)-\lambda_{n} J^{\prime}\left(w_{n}\right)
$$

for some sequence $\left(\lambda_{n}\right) \subset \mathbb{R}$. Since $w_{n} \in \mathcal{N}_{+}$, (3.7) implies

$$
\lambda_{n} J^{\prime}\left(w_{n}\right) w_{n} \rightarrow 0
$$

By Fatou's Lemma and $\left(f_{3}\right)$ we also have

$$
\begin{aligned}
\limsup _{n \in \mathbb{N}} J^{\prime}\left(w_{n}\right) w_{n} & =\limsup _{n \in \mathbb{N}} \int_{\mathbb{R}^{N}} f_{+}\left(w_{n}\right) w_{n}-f_{+}^{\prime}\left(w_{n}\right) w_{n}^{2} \mathrm{~d} x \\
& \leqslant \int_{\mathbb{R}^{N}} f_{+}(w) w-f_{+}^{\prime}(w) w^{2} \mathrm{~d} x<0,
\end{aligned}
$$

and hence (3.8) forces $\lambda_{n} \rightarrow 0$. Since $J^{\prime}\left(w_{n}\right)$ remains bounded in $E^{*}$, (3.7) implies that

$$
\Phi_{+}^{\prime}\left(w_{n}\right) \rightarrow 0 \text {. }
$$

In particular this implies that $\Phi^{\prime}(w)=\Phi_{+}^{\prime}(w)=0$, and hence $\Phi(w) \geqslant c_{+}$. In fact, by a standard decomposition method for Palais-Smale sequences of $\Phi_{+}$(see e.g. [15]), we now deduce that $w \in \mathcal{K}^{+}$and that $w_{n} \rightarrow w$ strongly in $E$. Thus $y_{n} * u_{n} \rightarrow w$ by (3.4) and (3.6), and hence

$$
\operatorname{dist}\left(u_{n}, \mathcal{K}^{+}\right) \leqslant\left\|\left(-y_{n}\right) * w-u_{n}\right\| \rightarrow 0
$$

as claimed.

We now put

$$
c_{0}=c_{+}+c_{-} .
$$

We also consider the set

$$
\mathcal{M}=\left\{u \in E \mid u^{+}, u^{-} \neq 0, \Phi^{\prime}(u) u^{+}=0=\Phi^{\prime}(u) u^{-}\right\},
$$

where $u^{+}=\max \{u, 0\}, u^{-}=\min \{u, 0\}$. Finally we introduce

$$
\mathcal{U}_{\varepsilon}=\left\{u \in E \mid u^{+} \in U_{\varepsilon}^{2}\left(\mathcal{K}^{+}\right), u^{-} \in U_{\varepsilon}^{2}\left(\mathcal{K}^{-}\right)\right\}
$$

and

$$
\mathcal{V}(\varepsilon, \delta)=\left(\mathcal{U}_{\varepsilon} \backslash \mathcal{U}_{\varepsilon / 2}\right) \cap \Phi^{c_{0}+\delta}
$$

for $\varepsilon, \delta>0$.

From now on we fix a generalized barycenter map $\beta=\beta_{2}$ on $L^{2}\left(\mathbb{R}^{N}\right)$ as constructed in Theorem 2.1. The main result of this section is the following. 
Proposition 3.3. There exists $\varepsilon>0$ and $\delta \in\left(0, \min \left\{c_{+}, c_{-}\right\}\right)$such that the following holds.

(i) $U_{\varepsilon}^{2}\left(\mathcal{K}^{+} \cup \mathcal{K}^{-}\right) \subset E \backslash\{0\}$, and $\beta\left(u^{+}\right) \neq \beta\left(u^{-}\right)$for every $u \in \mathcal{U}_{\varepsilon}$.

(ii) $\max _{i= \pm}\left(\left|\Phi^{\prime}(u) u^{i}\right| /\left\|u^{i}\right\|\right) \geqslant 6 \delta / \varepsilon$ for every $u \in \mathcal{V}(\varepsilon, \delta)$.

(iii) $\mathcal{M}^{c_{0}+\delta} \subset \operatorname{int}\left(\mathcal{U}_{\varepsilon / 2}\right)$.

(iv) There exist radially symmetric functions $w_{1}, w_{2} \in \mathcal{N}$ with compact support, and there exists $0<t_{0}<1$ satisfying:

$$
\begin{aligned}
& w_{1} \geqslant 0, \quad w_{2} \leqslant 0, \\
& (1+t) w_{1} \in U_{\varepsilon}^{2}\left(\mathcal{K}^{+}\right), \quad(1+t) w_{2} \in U_{\varepsilon}^{2}\left(\mathcal{K}^{-}\right) \quad \text { for }|t| \leqslant t_{0}, \\
& \Phi\left(w_{1}\right)<c_{+}+\frac{\delta}{4}, \quad \Phi\left(w_{2}\right)<c_{-}+\frac{\delta}{4}, \\
& \Phi\left(\left(1 \pm t_{0}\right) w_{1}\right) \leqslant c_{+}-2 \delta, \quad \Phi\left(\left(1 \pm t_{0}\right) w_{2}\right) \leqslant c_{-}-2 \delta .
\end{aligned}
$$

The remainder of this section is occupied with the proof of Proposition 3.3. We start with the following lemma.

Lemma 3.4. For $\varepsilon>0$ sufficiently small there holds

(i) $\inf _{u \in U_{\varepsilon}^{2}\left(\mathcal{K}^{+} \cup \mathcal{K}^{-}\right)}|u|_{2}^{2}>0$.

(ii) $\beta\left(u^{+}\right) \neq \beta\left(u^{-}\right)$for every $u \in \mathcal{U}_{\varepsilon}$.

Proof. (i) Since $\mathcal{K}_{\text {rad }}^{+} \cup \mathcal{K}_{\text {rad }}^{-}$is compact we have

$$
\inf _{u \in \mathcal{K}^{+} \cup \mathcal{K}^{-}}|u|_{2}^{2}=\inf _{u \in \mathcal{K}_{\text {rad }}^{+} \cup \mathcal{K}_{\text {rad }}^{-}}|u|_{2}^{2}>0 .
$$

This implies (i) for $\varepsilon>0$ sufficiently small.

(ii) Suppose by contradiction that there is $u_{n} \in \mathcal{U}_{1 / n}$ with $x_{n}:=\beta\left(u_{n}^{+}\right)=\beta\left(u_{n}^{-}\right) \in \mathbb{R}^{N}$ for $n \in \mathbb{N}$. After translations we may assume that $x_{n}=0$ for every $n$. Now we choose $v_{n} \in \mathcal{K}^{+}$with $\left|u_{n}^{+}-v_{n}\right|_{2}<\frac{2}{n}$ and set $y_{n}=-\beta\left(v_{n}\right)$. Then $y_{n} * v_{n} \in \mathcal{K}_{\text {rad }}^{+}$, and, passing to a subsequence if necessary, we may assume that $y_{n} * v_{n} \rightarrow \psi_{1} \in \mathcal{K}_{\text {rad }}^{+}$. This implies $y_{n} * u_{n}^{+} \rightarrow \psi_{1}$ in $L^{2}$, and therefore

$$
y_{n}=\beta\left(y_{n} * u_{n}^{+}\right) \rightarrow 0 \quad \text { as } n \rightarrow \infty .
$$

We conclude that $u_{n}^{+} \rightarrow \psi_{1}$ in $L^{2}$. Passing again to a subsequence, we can also achieve that $u_{n}^{-} \rightarrow \psi_{2} \in \mathcal{K}_{\text {rad }}^{-}$in $L^{2}$. Hence $u_{n} \rightarrow \psi_{1}+\psi_{2}$ in $L^{2}\left(\mathbb{R}^{N}\right)$, which implies that

$$
\left(\psi_{1}+\psi_{2}\right)^{+}=\lim _{n \rightarrow \infty} u_{n}^{+}=\psi_{1}
$$

and

$$
\left(\psi_{1}+\psi_{2}\right)^{-}=\lim _{n \rightarrow \infty} u_{n}^{-}=\psi_{2}
$$

This however contradicts the fact that $\psi_{1}$ is positive and $\psi_{2}$ is negative on all of $\mathbb{R}^{N}$.

Lemma 3.5. (i) Let $\left(u_{n}\right) \in E$ be a sequence such that $u_{n} \geqslant 0$ for all $n \in \mathbb{N}$,

$$
\inf _{n}\left\|u_{n}\right\|>0, \quad \lim \Phi^{\prime}\left(u_{n}\right) u_{n}=0 \quad \text { and } \quad \limsup \Phi\left(u_{n}\right) \leqslant c_{+} .
$$

Then $\Phi\left(u_{n}\right) \rightarrow c_{+}$and $\operatorname{dist}_{1}\left(u_{n}, \mathcal{K}^{+}\right) \rightarrow 0$ as $n \rightarrow \infty$. 
(ii) Let $\left(u_{n}\right) \in E$ be a sequence such that $u_{n} \leqslant 0$ for all $n \in \mathbb{N}$,

$$
\inf _{n}\left\|u_{n}\right\|>0, \quad \lim \Phi^{\prime}\left(u_{n}\right) u_{n}=0 \quad \text { and } \quad \limsup \Phi\left(u_{n}\right) \leqslant c_{-} .
$$

Then $\Phi\left(u_{n}\right) \rightarrow c_{-}$and $\operatorname{dist}_{1}\left(u_{n}, \mathcal{K}^{-}\right) \rightarrow 0$ as $n \rightarrow \infty$.

Proof. (i) It is sufficient to show that $\Phi\left(u_{n}\right) \rightarrow c_{+}$and $\operatorname{dist}_{1}\left(u_{n}, \mathcal{K}^{+}\right) \rightarrow 0$ hold for a subsequence. We put $\sigma_{n}:=$ $\Phi^{\prime}\left(u_{n}\right) u_{n}$, and we note that $\left(\mathrm{f}_{4}\right)$ yields

$$
\begin{aligned}
\left(\frac{1}{2}-\frac{1}{\theta}\right)\left\|u_{n}\right\|^{2} & \leqslant \Phi\left(u_{n}\right)-\frac{1}{\theta} \Phi^{\prime}\left(u_{n}\right) u_{n}+\int_{\mathbb{R}^{N}}\left(F\left(u_{n}\right)-\frac{1}{\theta} f\left(u_{n}\right) u_{n}\right) \mathrm{d} x \\
& \leqslant \Phi\left(u_{n}\right)-\frac{\sigma_{n}}{\theta} \leqslant c_{+}+o(1) .
\end{aligned}
$$

In particular $\left\|u_{n}\right\|$ remains bounded. Since furthermore $\inf _{n}\left\|u_{n}\right\|>0$, we may by a similar argument as in the proof of (3.6) assume that, translating suitably, for a subsequence we have

$$
u_{n} \rightarrow u \in E \backslash\{0\} .
$$

By $\left(f_{3}\right)$,

$$
\kappa_{t}:=\int_{\mathbb{R}^{N}}\left[f^{\prime}(t u) u^{2}-\frac{1}{t} f(t u) u\right] \mathrm{d} x>0 \text { for } t>0 .
$$

For $n \in \mathbb{N}$ we define $g_{n} \in C^{1}((0, \infty))$ by $g_{n}(t)=\Phi^{\prime}\left(t u_{n}\right) u_{n}$. Then $g_{n}^{\prime}(t)=\Phi^{\prime \prime}\left(t u_{n}\right)\left(u_{n}, u_{n}\right)$ for $t>0$. Moreover we set

$$
h_{n}(t)=\int_{\mathbb{R}^{N}}\left[f\left(u_{n}\right) u_{n}-\frac{1}{t} f\left(t u_{n}\right) u_{n}\right] \mathrm{d} x, \quad \text { for } n \in \mathbb{N}, t>0 .
$$

Since the functional $w \mapsto \int_{\mathbb{R}^{N}}\left[f^{\prime}(t w) w^{2}-\frac{1}{t} f(t w) w\right] \mathrm{d} x$ is weakly lower semicontinuous by virtue of $\left(\mathrm{f}_{3}\right)$ and Fatou's Lemma, we obtain

$$
\begin{aligned}
g_{n}^{\prime}(t)=\Phi^{\prime \prime}\left(t u_{n}\right)\left(u_{n}, u_{n}\right) & =\left\|u_{n}\right\|^{2}-\int_{\mathbb{R}^{N}} f^{\prime}\left(t u_{n}\right) u_{n}^{2} \mathrm{~d} x=\sigma_{n}+\int_{\mathbb{R}^{N}} f\left(u_{n}\right) u_{n}-f^{\prime}\left(t u_{n}\right) u_{n}^{2} \mathrm{~d} x \\
& =o(1)+\int_{\mathbb{R}^{N}}\left[\frac{1}{t} f\left(t u_{n}\right) u_{n}-f^{\prime}\left(t u_{n}\right) u_{n}^{2}\right] \mathrm{d} x+h_{n}(t) \\
& \leqslant o(1)+\int_{\mathbb{R}^{N}}\left[\frac{1}{t} f(t u) u-f^{\prime}(t u) u^{2}\right] \mathrm{d} x+h_{n}(t)=o(1)-\kappa_{t}+h_{n}(t)
\end{aligned}
$$

as $n \rightarrow \infty$. We claim that

$$
h_{n}(t) \rightarrow 0 \quad \text { as } t \rightarrow 1 \text { uniformly in } n \in \mathbb{N} .
$$

Indeed, there are constants $C_{i}>0$ such that for $n \in \mathbb{N}, t \in\left[\frac{1}{2}, \frac{3}{2}\right]$ we have

$$
\begin{aligned}
\left|h_{n}(t)\right| & =\left|\int_{\mathbb{R}^{N}}\left[f\left(u_{n}\right) u_{n}-\frac{1}{t} f\left(t u_{n}\right) u_{n}\right] \mathrm{d} x\right| \\
& \leqslant\left|\int_{\mathbb{R}^{N}}\left[f\left(u_{n}\right) u_{n}-f\left(t u_{n}\right) t u_{n}\right] \mathrm{d} x\right|+\left|t-\frac{1}{t}\right|\left|\int_{\mathbb{R}^{N}} f\left(t u_{n}\right) u_{n} \mathrm{~d} x\right|
\end{aligned}
$$




$$
\begin{aligned}
& =\left|\int_{\mathbb{R}^{N}} \int_{t}^{1}\left[f^{\prime}\left(s u_{n}\right) s u_{n}+f\left(s u_{n}\right)\right] u_{n} \mathrm{~d} s \mathrm{~d} x\right|+\left|t-\frac{1}{t}\right|\left|\int_{\mathbb{R}^{N}} f\left(t u_{n}\right) u_{n} \mathrm{~d} x\right| \\
& \leqslant C_{1} \int_{\mathbb{R}^{N}}\left|\int_{t}^{1}\left(\left|u_{n}\right|^{2}+\left|u_{n}\right|^{p}\right) \mathrm{d} s\right| \mathrm{d} x+C_{2}\left|t-\frac{1}{t}\right| \int_{\mathbb{R}^{N}}\left(\left|u_{n}\right|^{2}+\left|u_{n}\right|^{p}\right) \mathrm{d} x \\
& \leqslant C_{3}|t-1|\left(\left\|u_{n}\right\|^{2}+\left\|u_{n}\right\|^{p}\right)+C_{4}\left|t-\frac{1}{t}\right|\left(\left\|u_{n}\right\|^{2}+\left\|u_{n}\right\|^{p}\right) \\
& \leqslant C_{5}|t-1|+C_{6}\left|t-\frac{1}{t}\right| .
\end{aligned}
$$

Using (3.14) and (3.15), we find numbers $\bar{t} \in\left(0, \frac{1}{2}\right)$ and $n_{0} \in \mathbb{N}$ such that

$$
g_{n}^{\prime}(t)<-\frac{\kappa_{1}}{2}<0 \quad \text { for } t \in[1-\bar{t}, 1+\bar{t}], n \geqslant n_{0} .
$$

Hence, since $g_{n}(1)=\sigma_{n} \rightarrow 0$ as $n \rightarrow \infty$, for $n$ large enough there exist $t_{n} \in \mathbb{R},\left|1-t_{n}\right| \leqslant 2 \sigma_{n} / \kappa_{1}<\bar{t}$ with $g_{n}\left(t_{n}\right)=0$, that is $w_{n}:=t_{n} u_{n} \in \mathcal{N}$. Moreover,

$$
\begin{aligned}
\left|\Phi\left(u_{n}\right)-\Phi\left(w_{n}\right)\right| & \leqslant\left(1-t_{n}^{2}\right)\left\|u_{n}\right\|^{2}+\left|\int_{\mathbb{R}^{N}}\left[F\left(u_{n}(x)\right)-F\left(t_{n} u_{n}(x)\right)\right] \mathrm{d} x\right| \\
& \leqslant o(1)+\int_{\mathbb{R}^{N}}\left|\int_{t_{n}}^{1} f\left(s u_{n}\right) u_{n} \mathrm{~d} s\right| \mathrm{d} x \leqslant o(1)+C_{7} \int_{\mathbb{R}^{N}}\left|\int_{t_{n}}^{1}\left(\left|u_{n}\right|^{2}+\left|u_{n}\right|^{p}\right) \mathrm{d} s\right| \mathrm{d} x \\
& \leqslant o(1)+C_{8}\left|1-t_{n}\right|\left(\left\|u_{n}\right\|^{2}+\left\|u_{n}\right\|^{p}\right) \leqslant o(1)+C_{9}\left|1-t_{n}\right|=o(1) .
\end{aligned}
$$

Since $w_{n} \geqslant 0$ for every $n$, we have $\Phi\left(w_{n}\right) \geqslant c_{+}$, and hence we conclude that $\Phi\left(w_{n}\right) \rightarrow c_{+}$. Now Lemma 3.2 implies $\operatorname{dist}_{1}\left(w_{n}, \mathcal{K}^{+}\right) \rightarrow 0$, hence also $\operatorname{dist}_{1}\left(u_{n}, \mathcal{K}^{+}\right) \rightarrow 0$, as claimed.

The proof of (ii) is similar.

The following is a rather immediate consequence of Lemma 3.5.

Corollary 3.6. Let $\left(u_{n}\right) \in E$ be a sequence such that

$$
\inf _{n}\left\|u_{n}^{ \pm}\right\|>0, \quad \lim \Phi^{\prime}\left(u_{n}\right) u_{n}^{ \pm} \rightarrow 0 \quad \text { and } \quad \limsup \Phi\left(u_{n}\right) \leqslant c_{0} .
$$

Then $\operatorname{dist}_{1}\left(u_{n}^{ \pm}, \mathcal{K}^{ \pm}\right) \rightarrow 0$ as $n \rightarrow \infty$.

We now fix $\varepsilon>0$ such that Lemma 3.4 holds.

Lemma 3.7. For $\delta>0$ small enough we have:

(i) $\mathcal{M}^{c_{0}+\delta} \subset \operatorname{int}\left(\mathcal{U}_{\varepsilon / 2}\right)$;

(ii) $\inf _{u \in \mathcal{V}(\varepsilon, \delta)} \sup _{i= \pm}\left(\left|\Phi^{\prime}(u) u^{i}\right|\right) /\left\|u^{i}\right\| \geqslant 6 \delta / \varepsilon$.

Proof. (i) Note that if $u \in \mathcal{M}$, then $u^{ \pm} \in \mathcal{N}$, and therefore $\left\|u^{ \pm}\right\| \geqslant d^{ \pm}$. Hence (i) follows from Corollary 3.6.

(ii) Clearly it suffices to prove that

$$
\inf _{u \in \mathcal{V}(\varepsilon, \delta)} \sup _{i= \pm} \frac{\left|\Phi^{\prime}(u) u^{i}\right|}{\left\|u^{i}\right\|}>0 \quad \text { for } \delta>0 \text { small enough. }
$$


In order to see this, suppose to the contrary that there is a sequence $\left(u_{n}\right)_{n} \subset \mathcal{V}\left(\varepsilon, \frac{1}{n}\right)$ with

$$
\sup _{i= \pm} \frac{\left|\Phi^{\prime}\left(u_{n}\right) u_{n}^{i}\right|}{\left\|u_{n}^{i}\right\|} \rightarrow 0 .
$$

Since $u_{n}^{ \pm} \in U_{\varepsilon}^{2}\left(\mathcal{K}^{ \pm}\right)$, we have $\inf _{n}\left\|u_{n}^{ \pm}\right\|>0$ by Lemma 3.4(i). Hence Corollary 3.6 implies that $\operatorname{dist}_{1}\left(u_{n}^{ \pm}, \mathcal{K}^{ \pm}\right) \rightarrow$ 0 , contrary to the assumption that $u_{n} \notin \mathcal{U}_{\varepsilon / 2}$ for all $n \in \mathbb{N}$.

Lemma 3.8. There exists $\varepsilon_{0}>0, \eta_{0}>0$ and $0<t_{0}<1$ such that for every $w \in U_{\varepsilon_{0}}^{1}\left(\mathcal{K}^{ \pm}\right)$there holds:

$$
\begin{aligned}
& (1+t) w \in U_{\varepsilon}^{2}\left(\mathcal{K}^{ \pm}\right) \quad \text { for }|t| \leqslant t_{0}, \\
& \Phi\left(\left[1 \pm t_{0}\right] w\right) \leqslant \Phi(w)-\eta_{0} .
\end{aligned}
$$

Proof. Clearly one can choose $\varepsilon_{0}, t_{0}$ sufficiently small such that (3.18) holds for every $w \in U_{\varepsilon_{0}}^{1}\left(\mathcal{K}^{ \pm}\right)$. Now since $\mathcal{K}_{\text {rad }}^{+} \cup \mathcal{K}_{\text {rad }}^{-}$is compact, Lemma 3.1 yields

$$
\eta:=\max _{u \in \mathcal{K}^{+} \cup \mathcal{K}^{-}} \Phi^{\prime \prime}(u)(u, u)=\max _{u \in \mathcal{K}_{\mathrm{rad}}^{+} \cup \mathcal{K}_{\mathrm{rad}}^{-}} \Phi^{\prime \prime}(u)(u, u)<0 .
$$

Hence, making $t_{0}>0$ smaller if necessary, we can achieve that

$$
\Phi((1+t) u) \leqslant \Phi(u)-\frac{\eta}{3} t^{2} \quad \text { for }|t| \leqslant t_{0}, u \in \mathcal{K}^{+} \cup \mathcal{K}^{-} .
$$

Now making $\varepsilon_{0}>0$ smaller if necessary and using that $\Phi$ is continuous, we find that

$$
\Phi\left(\left(1 \pm t_{0}\right) w\right) \leqslant \Phi(w)-\frac{\eta}{4} t_{0}^{2} \quad \text { for } w \in U_{\varepsilon_{0}}^{1}\left(\mathcal{K}^{+} \cup \mathcal{K}^{-}\right) .
$$

Thus (3.19) holds with $\eta_{0}:=\frac{\eta}{4} t_{0}^{2}$.

We fix $\varepsilon_{0}, \eta_{0}$ and $t_{0}$ such that Lemma 3.8 holds.

Lemma 3.9. For $\delta>0$ small enough there exist radially symmetric functions $w_{1}, w_{2} \in \mathcal{N} \cap U_{\varepsilon_{0}}^{1}\left(\mathcal{K}^{+} \cup \mathcal{K}^{-}\right)$with compact support and such that (3.9)-(3.12) hold.

Proof. We first choose $\psi_{1} \in \mathcal{K}_{\text {rad }}^{+}, \psi_{2} \in \mathcal{K}_{\text {rad }}^{-}$. We also choose $\varphi \in C_{0}^{\infty}([0, \infty))$ with $0 \leqslant \varphi \leqslant 1, \varphi(t)=1$ for $t \leqslant 1$ and $\varphi(t)=0$ for $t \geqslant 2$. Now we define $\psi_{i, r} \in E, i=1,2$, by

$$
\psi_{i, r}(x)=\psi_{i}(x) \varphi(r|x|), \quad x \in \mathbb{R}^{N} .
$$

It is easy to see that $\psi_{i, r} \rightarrow \psi_{i}$ in $E$ as $r \rightarrow 0$. Now let $\rho: E \backslash\{0\} \rightarrow \mathcal{N}$ denote the radial projection on the Nehari manifold $\mathcal{N}$, and set $w_{i, r}:=\rho\left(\psi_{i, r}\right) \in \mathcal{N}, i=1,2$. Then $w_{i, r} \rightarrow \psi_{i}$ in $E$ as $r \rightarrow \infty$. Hence there exists $r_{0}$ such that

$$
w_{1, r} \in U_{\varepsilon_{0}}^{1}\left(\mathcal{K}^{+}\right), \quad w_{2, r} \in U_{\varepsilon_{0}}^{1}\left(\mathcal{K}^{-}\right)
$$

for $r>r_{0}$. Now we consider

$$
\delta \in\left(0, \frac{\eta_{0}}{3}\right),
$$

and fix $r>r_{0}$ such that

$$
\Phi\left(w_{1, r}\right)<c_{+}+\frac{\delta}{4}, \quad \Phi\left(w_{2, r}\right)<c_{-}+\frac{\delta}{4} .
$$


Setting $w_{i}:=w_{i, r}$ the conditions (3.9)-(3.11) are satisfied. Moreover it follows from (3.20)-(3.22) and Lemma 3.8 that

$$
\Phi\left(\left(1 \pm t_{0}\right) w_{1}\right) \leqslant \Phi\left(w_{1}\right)-\eta_{0}<c_{+}-2 \delta .
$$

In the same way we deduce $\Phi\left(\left(1 \pm t_{0}\right) w_{2}\right)<c_{-}-2 \delta$, hence (3.12) is also satisfied. This finishes the proof.

The proof of Proposition 3.3 is completed by combining Lemmas 3.4, 3.7-3.9.

\section{The equation on a bounded domain}

We now consider a bounded domain $\Omega \subset \mathbb{R}^{N}$, and we set $H=H_{0}^{1}(\Omega)$. We regard $H$ as a closed subspace of $E$, hence $H$ is endowed with the scalar product $(\cdot, \cdot)$ and the norm $\|\cdot\|$ induced by the embedding $H \subset E$. Weak solutions $u \in H$ of (1.2) are critical points of the functional $\Psi=\left.\Phi\right|_{H} \in C^{2}(H)$. It is well known (cf. [1]) that $\Psi$ satisfies the Palais-Smale condition. We fix $a_{0}<0$, and we choose a Lipschitz continuous function $\chi: \mathbb{R} \rightarrow \mathbb{R}$ such that $0 \leqslant \chi \leqslant 1, \chi(s)=1$ for $s \geqslant a_{0}$ and $\chi(s)=0$ for $s \leqslant a_{0}-1$. Then there is a flow $\varphi: \mathcal{D} \subset \mathbb{R} \times H \rightarrow H$ satisfying

$$
\left\{\begin{array}{l}
\frac{\mathrm{d}}{\mathrm{d} t} \varphi(t, u)=-\chi(\Psi(\varphi(t, u))) \nabla \Psi(\varphi(t, u)), \\
\varphi(0, u)=u .
\end{array}\right.
$$

Here $\mathcal{D}=\left\{(t, u): u \in H, t \in\left(T^{-}(u), \infty\right)\right\}$, where $T^{-}(u)<0$ is the maximal existence time of the trajectory $t \mapsto \varphi(t, u)$ in negative direction (note that by construction the flow exists for all positive times). We will frequently write $\varphi^{t}$ in place of $\varphi(t, \cdot)$. We furthermore introduce

$$
\mathcal{N}_{\Omega}=\mathcal{N} \cap H, \quad \mathcal{M}_{\Omega}=\mathcal{M} \cap H
$$

and

$$
\bar{c}:=\inf \Psi\left(\mathcal{N}_{\Omega}\right), \quad c_{1}:=\inf \Psi\left(\mathcal{M}_{\Omega}\right) .
$$

Note that

$$
c_{1} \geqslant c_{0}=c_{+}+c_{-}>\min \left\{c_{+}, c_{-}\right\}=\min \Phi(\mathcal{N})
$$

(see Lemma 3.1(v)). Since $\Psi^{\prime}(u) u^{+}=0=\Psi^{\prime}(u) u^{-}$for any critical point $u$ of $\Psi$, all sign changing critical points are contained in $\mathcal{M}_{\Omega}$. We also have

Proposition 4.1. (i) The infimum $c_{1}$ is attained in $\mathcal{M}_{\Omega}$, and every $u \in \mathcal{M}_{\Omega}^{c_{1}}$ is a sign changing critical point of $\Psi$.

(ii) If $u \in \mathcal{M}_{\Omega}^{c_{1}}$ is isolated among all sign changing critical points, then the critical groups of $u$ satisfy $\mathcal{C}_{k}(u, \Psi) \cong$ $\delta_{k 2} \mathbb{Z}$ for $k \in \mathbb{Z}$.

(iii) Every sign changing critical point with $\Psi(u)<c_{1}+\bar{c}$ has precisely two nodal domains.

We recall that the critical groups of a critical point $u$ of $\Psi$ with $\Psi(u)=c$ are defined by

$$
C_{k}(u, \Psi):=H_{k}\left(\Psi^{c}, \Psi^{c} \backslash\{u\}\right), \quad k \in \mathbb{Z},
$$

where $H_{*}$ denotes singular homology with coefficients in $\mathbb{Z}$. Proposition 4.1(i) has been proved under slightly stronger hypotheses in [10], see also [14]. For the proof of Proposition 4.1 we require the following technical lemma. 
Lemma 4.2. For every $u \in \mathcal{M}_{\Omega}$ there exists a continuous function $\tau_{u}: H \rightarrow[0, \infty)^{2}$ such that

$$
\begin{array}{ll}
\tau_{u}\left(s u^{+}+t u^{-}\right)=(s, t) & \text { for } s, t \geqslant 0, \\
\tau_{u}(v)=(1,1) & \text { if and only if } v \in \mathcal{M}_{\Omega} .
\end{array}
$$

Proof. We first define $\sigma: H \rightarrow[0, \infty)$ by

$$
\sigma(v)= \begin{cases}\frac{\int_{\Omega} f\left(v^{+}\right) v^{+}}{\left\|v^{+}\right\|^{2}}, & v^{+} \neq 0 \\ 0, & v^{+}=0 .\end{cases}
$$

Note that $\sigma$ is continuous, as follows easily from $\left(\mathrm{f}_{1}\right),\left(\mathrm{f}_{2}\right)$ and Sobolev embeddings. Moreover

$$
\sigma(v)=1 \quad \text { if and only if } \quad v^{+} \in \mathcal{N} .
$$

Now we fix $u \in \mathcal{M}_{\Omega}$. Then

$$
s \mapsto \xi(s):=\sigma\left(s u^{+}\right)
$$

is strictly increasing on $[0, \infty)$ by virtue of $\left(\mathrm{f}_{3}\right)$, and $\xi(s) \rightarrow \infty$ for $s \rightarrow \infty$. Hence $\xi^{-1} \in C([0, \infty),[0, \infty))$ exists and is strictly increasing. Now we define

$$
\tau_{+}: H \rightarrow[0, \infty), \quad \tau_{+}(v)=\xi^{-1}(\sigma(v)) .
$$

Then $\tau_{+}\left(s u^{+}+t u^{-}\right)=s$ for $s, t \geqslant 0$, and for $v \in H$ there holds $\tau_{+}(v)=1$ if and only if $v^{+} \in \mathcal{N}$.

In a similar way we construct a continuous function $\tau_{-}: H \rightarrow[0, \infty)$ such that $\tau_{-}\left(s u^{+}+t u^{-}\right)=t$ for $s, t \geqslant 0$, and $\tau_{-}(v)=1$ if and only if $v^{-} \in \mathcal{N}$. We then set $\tau_{u}(v)=\left(\tau_{+}\left(v^{+}\right), \tau_{-}\left(v^{-}\right)\right)$, and we conclude that the thus defined map $\tau_{u}: H \rightarrow[0, \infty)^{2}$ has the asserted properties.

Proof of Proposition 4.1. (i) Consider a sequence $\left(u_{n}\right) \subset \mathcal{M}_{\Omega}$ with $\Psi\left(u_{n}\right) \rightarrow c_{1}$. Since

$$
\left(\frac{1}{2}-\frac{1}{\theta}\right)\left\|u_{n}\right\|^{2} \leqslant \Psi\left(u_{n}\right)
$$

for every $n$ by (3.2), the sequences $\left(u_{n}^{ \pm}\right)$are bounded in $H$. Passing to a subsequence, there are $u_{+}, u_{-} \in H$ such that $u_{n}^{+} \rightarrow u_{+}, u_{n}^{-} \rightarrow u_{+}$as $n \rightarrow \infty$. Hence $u_{n}^{ \pm} \rightarrow u_{ \pm}$strongly in $L^{s}(\Omega)$ for $1 \leqslant s<2^{*}$, which in particular implies that

$$
u_{+} \geqslant 0, \quad u_{-} \leqslant 0, \quad u_{+} \cdot u_{-}=0 \quad \text { a.e. in } \Omega .
$$

Using ( $\left.f_{2}\right)$ and Lemma 3.1(iv) we also find

$$
d_{ \pm}^{2} \leqslant \lim _{n \rightarrow \infty}\left\|u_{n}^{ \pm}\right\|^{2}=\lim _{n \rightarrow \infty} \int_{\Omega} f\left(u_{n}^{ \pm}\right) u_{n}^{ \pm}=\int_{\Omega} f\left(u_{ \pm}\right) u_{ \pm}
$$

and

$$
\lim _{n \rightarrow \infty} \int_{\Omega} F\left(u_{n}^{ \pm}\right)=\int_{\Omega} F\left(u_{ \pm}\right) .
$$

From (4.6) we infer that $u_{ \pm} \neq 0$, and that

$$
\left\|u_{ \pm}\right\|^{2} \leqslant \int_{\Omega} f\left(u_{ \pm}\right) u_{ \pm}
$$


by the weak lower semicontinuity of $\|\cdot\|$. Hence there are $t_{ \pm} \in(0,1]$ with

$$
\left\|t_{ \pm} u_{ \pm}\right\|^{2}=\int_{\Omega} f\left(t_{ \pm} u_{ \pm}\right) t u_{ \pm} .
$$

Using (4.6), (4.7) and the fact that the function $t \mapsto \frac{1}{2} f(t) t-F(t)$ is increasing on $(0, \infty)$ by ( $\mathrm{f}_{3}$ ), we find

$$
\begin{aligned}
\Psi\left(t_{ \pm} u_{ \pm}\right) & =\frac{1}{2} \int_{\Omega} f\left(t_{ \pm} u_{ \pm}\right) t_{ \pm} u_{ \pm}-\int_{\Omega} F\left(t_{ \pm} u_{ \pm}\right) \leqslant \frac{1}{2} \int_{\Omega} f\left(u_{ \pm}\right) u_{ \pm}-\int_{\Omega} F\left(u_{ \pm}\right) \\
& =\lim _{n \rightarrow \infty}\left(\frac{1}{2} \int_{\Omega} f\left(u_{n}^{ \pm}\right) u_{n}^{ \pm}-\int_{\Omega} F\left(u_{n}^{ \pm}\right)\right)=\lim _{n \rightarrow \infty} \Psi\left(u_{n}^{ \pm}\right) .
\end{aligned}
$$

Moreover, by (4.5) and (4.9) the function $u=t_{+} u_{+}+t_{-} u_{-}$is an element of $\mathcal{M}$, and

$$
\Psi(u)=\Psi\left(t_{+} u_{+}+t_{-} u_{-}\right) \leqslant \lim _{n \rightarrow \infty}\left(\Psi\left(u_{n}^{+}\right)+\Psi\left(u_{n}^{-}\right)\right)=\lim _{n \rightarrow \infty} \Psi\left(u_{n}\right)=c_{1} .
$$

Hence $u$ is a minimizer for $\Psi$ on $\mathcal{M}$, that is, $\mathcal{M}_{\Omega}^{c_{1}}$ is non-empty.

Next let $u \in \mathcal{M}_{\Omega}^{c_{1}}$ arbitrary. Fix $s_{0} \in(0,1)$ and define

$$
p\left(s_{1}, s_{2}\right):=s_{1} u^{+}+s_{2} u^{-} \text {for } s_{1}, s_{2} \in I_{0}:=\left[1-s_{0}, 1+s_{0}\right] .
$$

Then $\tau_{u} \circ p: I_{0}^{2} \rightarrow \mathbb{R}^{2}$ is just the inclusion, in particular

$$
\operatorname{deg}\left(\tau_{u} \circ p, I_{0}^{2},(1,1)\right)=1 .
$$

By Lemma 3.1(ii) we have

$$
\Psi\left(p\left(s_{1}, s_{2}\right)\right)<c_{1} \quad \text { for }\left(s_{1}, s_{2}\right) \in I_{0}^{2} \backslash\{(1,1)\} .
$$

Since moreover $\Psi$ is nonincreasing along trajectories of $\varphi$, we infer

$$
\varphi^{t} \circ p\left(\partial I_{0}^{2}\right) \cap \mathcal{M}_{\Omega}=\varnothing \quad \text { for every } t \geqslant 0,
$$

which by (4.3) implies

$$
\tau_{u} \circ \varphi^{t} \circ p\left(s_{1}, s_{2}\right) \neq(1,1) \text { for every }\left(s_{1}, s_{2}\right) \in \partial I_{0}^{2}, t \geqslant 0 .
$$

Using the homotopy invariance of the degree we conclude that

$$
\operatorname{deg}\left(\tau_{u} \circ \varphi^{t} \circ p, I_{0}^{2},(1,1)\right)=1 \text { for every } t \geqslant 0
$$

Hence there exists $\left(\overline{s_{1}}, \overline{s_{2}}\right) \in \operatorname{int}\left(I_{0}^{2}\right)$ with $\tau_{u} \circ \varphi^{1} \circ p\left(\overline{s_{1}}, \overline{s_{2}}\right)=(1,1)$, that is, $\bar{u}:=\varphi^{1} \circ p\left(\overline{s_{1}}, \overline{s_{2}}\right) \in \mathcal{M}_{\Omega}$. In particular $\Psi(\bar{u}) \geqslant c_{1}$. In fact, (4.11) now forces

$$
\bar{u}=\varphi^{1}(u) \quad \text { and } \quad \Psi(\bar{u})=\Psi\left(\varphi^{1}(u)\right)=c_{1} .
$$

This implies that

$$
\left\|\Psi^{\prime}(u)\right\|^{2}=-\left.\frac{\partial}{\partial t}\right|_{t=0} \Psi\left(\varphi^{t}(u)\right)=0,
$$

hence $u$ is a critical point of $\Psi$.

(ii) Consider an isolated critical point $u \in \mathcal{M}^{c_{1}}$ and a neighborhood $N \subset H$ of $u$ containing no other sign changing critical point. Hence $N \cap \mathcal{M}_{\Omega}^{c_{1}}=\{u\}$ by (i). In view of (4.3) we may consider $\tau_{u}$ as a map of pairs

$$
\left(\Psi^{c_{1}} \cap N, \Psi^{c_{1}} \cap N \backslash\{u\}\right) \rightarrow\left([0, \infty)^{2},[0, \infty)^{2} \backslash\{(1,1)\}\right) .
$$


Now choose $s_{0} \in(0,1)$ small enough so that

$$
p(s, t) \in N \text { for } s, t \in I_{0},
$$

where $I_{0}$ and $p$ are defined as in (4.10). Observe that $p$ may be viewed as a map of pairs

$$
\left(I_{0}^{2}, I_{0}^{2} \backslash\{(1,1)\}\right) \stackrel{p}{\longrightarrow}\left(\Psi^{c_{1}} \cap N, \Psi^{c_{1}} \cap N \backslash\{u\}\right)
$$

and that

$$
\tau_{u} \circ p:\left(I_{0}^{2}, I_{0}^{2} \backslash\{(1,1)\}\right) \rightarrow\left([0, \infty)^{2},[0, \infty)^{2} \backslash\{(1,1)\}\right)
$$

is the inclusion. Hence

$$
\tau_{u *} \circ p_{*}: H_{2}\left(I_{0}^{2}, I_{0}^{2} \backslash\{(1,1)\}\right) \rightarrow H_{2}\left([0, \infty)^{2},[0, \infty)^{2} \backslash\{(1,1)\}\right) \cong \mathbb{Z}
$$

is an isomorphism, which implies that

$$
\mathcal{C}_{2}(u, \Psi)=H_{2}\left(\Psi^{c_{1}}, \Psi^{c_{1}} \backslash\{u\}\right) \cong H_{2}\left(\Psi^{c_{1}} \cap N, \Psi^{c_{1}} \cap N \backslash\{u\}\right)
$$

is nontrivial.

Next we observe that the Morse index of $u$ is at least 2. In fact this holds for any sign changing critical point $u$ of $\Psi$, since by Lemma 3.1(i) we have

$$
\Psi^{\prime \prime}(u)\left(s_{1} u^{+}+s_{2} u^{-}, s_{1} u^{+}+s_{2} u^{-}\right)=s_{1}^{2} \Psi^{\prime \prime}\left(u^{+}\right)\left(u^{+}, u^{+}\right)+s_{2}^{2} \Psi^{\prime \prime}\left(u^{-}\right)\left(u^{-}, u^{-}\right)<0
$$

for every $\left(s_{1}, s_{2}\right) \in \mathbb{R}^{2} \backslash\{0\}$. The claim now follows from [3, Proposition 3.3].

(iii) We note that every critical point $u$ of $\Psi$ is a continuous function on $\Omega$. Hence, if $\Omega^{*}$ is a nodal domain of $u$, then $u \chi_{\Omega^{*}}$ defines an element of $H$ by [23, Lemma 1]; here $\chi_{\Omega^{*}}$ denotes the characteristic function of $\Omega^{*}$. Moreover, since

$$
\Psi^{\prime}\left(u \chi \Omega^{*}\right) u \chi \Omega_{\Omega^{*}}=\Psi^{\prime}(u) u \chi \Omega_{\Omega^{*}}=0,
$$

we have $\Psi\left(u \chi_{\Omega^{*}}\right) \geqslant \bar{c}$. Now suppose that $u$ has three nodal domains $\Omega_{1}, \Omega_{2}, \Omega_{3}$ such that $u>0$ on $\Omega_{1}$ and $u<0$ on $\Omega_{2}$. Then $u \chi \Omega_{\Omega_{1} \cup \Omega_{2}} \in \mathcal{M}_{\Omega}$ and $c_{1} \leqslant \Psi\left(u \chi \chi_{\Omega_{1} \cup \Omega_{2}}\right)$. Hence

$$
c_{1}+\bar{c} \leqslant \Psi\left(u \chi_{\Omega_{1} \cup \Omega_{2}}\right)+\Psi\left(u \chi_{\Omega_{3}}\right) \leqslant \Psi(u),
$$

which shows the assertion.

Next we consider the cones

$$
H^{+}=\{u \in H \mid u \geqslant 0\}, \quad H^{-}=\{u \in H \mid u \leqslant 0\},
$$

the closed convex sets

$$
D_{\alpha}^{ \pm}=\left\{u \in H \mid \operatorname{dist}_{1}\left(u, H^{ \pm}\right) \leqslant \alpha\right\}
$$

and the set $D_{\alpha}=D_{\alpha}^{+} \cup D_{\alpha}^{-}$for $\alpha>0$. Moreover, we call a closed subset $\mathcal{E} \subset H$ with nonempty interior strictly positively invariant under the flow $\varphi$ if $\varphi^{t}(u) \in \operatorname{int}(\mathcal{E})$ for every $u \in \mathcal{E}, t>0$.

Proposition 4.3. For $\alpha>0$ small enough there holds

(i) $D_{\alpha} \cap \mathcal{M}_{\Omega}=\varnothing$.

(ii) $\partial D_{\alpha}$ contains no critical points of $\Psi$.

(iii) For every $b \in\left[0, c_{1}\right)$ the set $D_{\alpha} \cup \Psi^{b}$ is strictly positively invariant under $\varphi$. 
Proof. (i) For $u \in \mathcal{M}_{\Omega}$ we have by (f $\mathrm{f}_{2}$ ), Lemma 3.1(iv) and Sobolev embeddings

$$
\begin{aligned}
0<\left(d_{+}\right)^{2} & \leqslant\left\|u^{+}\right\|^{2}=\int_{\Omega} f\left(u^{+}\right) u^{+} \leqslant C\left(\left|u^{+}\right|_{2}^{2}+\left|u^{+}\right|_{p}^{p}\right) \leqslant C \inf _{v \in-\mathcal{P}}\left(|u-v|_{2}^{2}+|u-v|_{p}^{p}\right) \\
& \leqslant C_{1} \inf _{v \in-\mathcal{P}}\left(\|u-v\|^{2}+\|u-v\|^{p}\right),
\end{aligned}
$$

and similarly

$$
0<\left(d_{-}\right)^{2} \leqslant C_{1} \inf _{v \in \mathcal{P}}\left(\|u-v\|^{2}+\|u-v\|^{p}\right),
$$

where $C_{1}>0$ is a constant. Hence $D_{\alpha} \cap \mathcal{M}_{\Omega}=\varnothing$ for $\alpha^{2}+\alpha^{p}<\frac{1}{C_{1}} \min \left\{\left(d_{+}\right)^{2},\left(d_{-}\right)^{2}\right\}$.

(ii) The gradient of $\Psi$ has the form $\nabla \Psi=\operatorname{Id}_{H}-A$ with $A: H \rightarrow H$ given by $A(u):=(-\Delta+1)^{-1} f(u)$ for $u \in H$. In other words, $v:=A(u)$ is uniquely determined by the relation

$$
\int_{\mathbb{R}^{n}}(\nabla v \nabla w+v w)=\int_{\mathbb{R}^{n}} f(u) w \quad \text { for all } w \in H .
$$

Now the same argument as in [4, Lemma 3.1] yields that

$$
A\left(\partial D_{\alpha}^{ \pm}\right) \subset \operatorname{int}\left(D_{\alpha}^{ \pm}\right),
$$

for $\alpha>0$ sufficiently small, which in particular implies that $\partial D_{\alpha}$ contains no fixed points of $A$, that is, no critical points of $\Psi$.

(iii) It suffices to prove the following:

$$
\left\{\begin{array}{l}
\text { For every } u \in \partial\left(D_{\alpha} \cup \Psi^{b}\right) \text { there is } \varepsilon>0 \text { such that } \\
\varphi^{t}(u) \in D_{\alpha} \cup \Psi^{b} \text { for } t \in[0, \varepsilon) \text { and } \varphi^{t}(u) \notin D_{\alpha} \cup \Psi^{b} \text { for } t \in(-\varepsilon, 0) .
\end{array}\right.
$$

Consider first $u \in \partial \Psi^{b} \backslash D_{\alpha}$. Then $\Psi(u)=b$ and hence $\chi(\Psi(u))=1$. Moreover, $u$ changes sign, and since $b<c_{1}$, $u$ is not a critical point of $\Psi$. Therefore

$$
\left.\frac{\partial}{\partial t} \Psi\left(\varphi^{t}(u)\right)\right|_{t=0}=-\|\nabla \Psi(u)\|^{2}<0,
$$

and thus there is $\varepsilon>0$ such that $\Psi\left(\varphi^{t}(u)\right)<b<\Psi\left(\varphi^{-t}(u)\right)$ for $t \in(0, \varepsilon)$. Making $\varepsilon>0$ smaller if necessary, we may also assume that $\varphi^{t}(u) \notin D_{\alpha}$ for $t \in(-\varepsilon, 0)$. Hence (4.16) holds in this case.

Now let $u \in \partial D_{\alpha}^{+}$with $\Psi(u) \geqslant b$. Then $\chi(\Psi(u))=1$ and consequently

$$
u+\lambda(-\chi(\Psi(u)) \nabla \Psi(u))=u+\lambda(-\nabla \Psi(u))=(1-\lambda) u+\lambda A(u) \in D_{\alpha}^{+}
$$

for $\lambda \in[0,1]$ by (4.15) and the convexity of $D_{\alpha}^{+}$. Since this is also true for $\tilde{u} \in D_{\alpha}^{+}$close to $u$, an application of [16, Theorem 4.1] yields that $\varphi^{t}(u) \in D_{\alpha}^{+}$for sufficiently small $t>0$. Moreover, by Mazur's separation theorem, there exists a continuous linear functional $j \in H^{*}$ and $\zeta \in \mathbb{R}$ such that $j(u)=\zeta$ and $j(w)>\zeta$ for $w \in \operatorname{int}\left(D_{\alpha}^{+}\right)$. Hence

$$
\left.\frac{\partial}{\partial t} j\left(\varphi^{t}(u)\right)\right|_{t=0}=j(-\nabla \Psi(u))=j(A(u)-u)=j(A(u))-\zeta>0
$$

by (4.15). From this we infer that $j\left(\varphi^{t}(u)\right)<\zeta$ and $\Psi\left(\varphi^{t}(u)\right)>b$ for $t \in(-\varepsilon, 0)$ provided that $\varepsilon$ is sufficiently small. Hence $\varphi^{t}(u) \notin D_{\alpha}^{+} \cup \Psi^{b}$ for $t \in(-\varepsilon, 0)$. A similar argument shows that for $u \in \partial D_{\alpha}^{-}$with $\Psi(u) \geqslant b$ there is $\varepsilon>0$ such that $\varphi^{t}(u) \in D_{\alpha}^{-}$for $t \in[0, \varepsilon)$ and $\varphi^{t}(u) \notin D_{\alpha}^{-} \cup \Psi^{b}$ for $t \in(-\varepsilon, 0)$. This completes the proof of (4.16). 


\section{Proof of Theorem 1.2}

Let $\varepsilon, \delta, t_{0}>0$ and $w_{1}, w_{2} \in E$ be given as in Proposition 3.3, and recall the values $c_{ \pm}, c_{0}$ and the set $\mathcal{U}_{\varepsilon}=\left\{u \in E \mid u^{+} \in U_{\varepsilon}^{2}\left(\mathcal{K}^{+}\right), u^{-} \in U_{\varepsilon}^{2}\left(\mathcal{K}^{-}\right)\right\}$defined in Section 3. Without loss of generality, we may assume that

$$
c_{+} \leqslant c_{-} .
$$

We choose $R>0$ so that $B_{R / 2}(0)$ contains the support of $w_{1}$ and $w_{2}$, and we suppose that $\Omega$ is a bounded domain containing $B_{5 R / 2}(0) \subset \Omega$. As in Section 4 we put $H=H_{0}^{1}(\Omega)$, and we consider the functional $\Psi$, the sets $\mathcal{N}_{\Omega}, \mathcal{M}_{\Omega}$, the values $\bar{c}, c_{1}$ and the flow $\varphi^{t}$ defined in this section. Since $B_{5 R / 2}(0) \subset \Omega$ and $B_{R / 2}(0)$ contains the support of $w_{1}$ and $w_{2}$,

$$
y * w_{1}+w_{2} \in \mathcal{M}_{\Omega} \quad \text { for every } y \in S_{R}:=\partial B_{R}(0),
$$

and together with (3.11) this implies that

$$
c_{1}<c_{0}+\frac{\delta}{2} \text {. }
$$

We need a variant of Lemma 4.2.

Lemma 5.1. There exists $\tau_{0}: H \rightarrow[0, \infty)^{2}$ such that

$$
\begin{array}{ll}
\tau_{0}\left(s\left(y * w_{1}\right)+t w_{2}\right)=(s, t) & \text { for every } s, t \geqslant 0, y \in S_{R}, \\
\tau_{0}(v)=(1,1) & \text { if and only if } v \in \mathcal{M}_{\Omega} .
\end{array}
$$

Proof. We consider the maps

$$
\sigma_{ \pm}: H \rightarrow[0, \infty), \quad \sigma_{ \pm}(v)= \begin{cases}\frac{\int_{\Omega} f\left(v^{ \pm}\right) v^{ \pm}}{\left\|v^{ \pm}\right\|^{2}}, & v^{ \pm} \neq 0 \\ 0, & v^{ \pm}=0\end{cases}
$$

as in the proof of Lemma 4.2 and observe that $\xi_{ \pm}(s):=\sigma_{ \pm}\left(s\left[y * w_{1}+w_{2}\right]\right)$ is independent of $y \in S_{R}$. Now we can define $\tau_{0}(v)=\left(\xi_{+}^{-1}\left(\sigma\left(v^{+}\right)\right), \xi_{-}^{-1}\left(\sigma\left(v^{-}\right)\right)\right)$as in the proof of Lemma 4.2.

Next we fix $\alpha>0$ such that Proposition 4.3 holds.

Lemma 5.2. There exists a number $T>0$ such that $\varphi^{T}(u) \in \operatorname{int}\left(D_{\alpha} \cup \Psi^{c_{0}-\delta}\right)$ for all $u \in \Psi^{c_{0}+\delta} \backslash \operatorname{int}\left(\mathcal{U}_{\varepsilon} \cap H\right)$.

Proof. Since $\mathcal{M}_{\Omega} \subset \mathcal{M}$ contains all sign changing critical points of $\Psi$, Proposition 3.3(iii) implies that the closed set $\Psi^{c_{0}+\delta} \backslash \operatorname{int}\left(\left(\mathcal{U}_{\varepsilon / 2} \cap H\right) \cup D_{\alpha}\right)$ contains no critical point of $\Psi$. Since $\Psi$ satisfies the Palais-Smale condition, we infer that

$$
\tau_{0}:=\inf \left\{\left\|\Psi^{\prime}(u)\right\|: u \in \Psi^{c_{0}+\delta} \backslash \operatorname{int}\left(\left(\mathcal{U}_{\varepsilon / 2} \cap H\right) \cup D_{\alpha} \cup \Psi^{c_{0}-\delta}\right)\right\}>0 .
$$

We also note that Proposition 3.3(ii) implies

$$
\left\|\Psi^{\prime}(u)\right\| \geqslant \max _{i= \pm} \frac{\Phi^{\prime}(u) u^{i}}{\left\|u^{i}\right\|} \geqslant \frac{6 \delta}{\varepsilon} \quad \text { for every } u \in \mathcal{V}(\varepsilon, \delta) \cap H=\left(\mathcal{U}_{\varepsilon} \backslash \mathcal{U}_{\varepsilon / 2}\right) \cap \Psi^{c_{0}+\delta} .
$$

Now we set $T:=3 \delta / \tau_{0}^{2}$, and we consider $u \in \Psi^{c_{0}+\delta} \backslash \operatorname{int}\left(\mathcal{U}_{\varepsilon} \cap H\right)$. By Proposition 4.3(iii) it suffices to show that

$$
\varphi^{t}(u) \in \operatorname{int}\left(D_{\alpha} \cup \Psi^{c_{0}-\delta}\right) \quad \text { for some } t \in[0, T] .
$$

We suppose by contradiction that this is false. Then one of the following two cases occurs. 
Case 1: $\varphi^{\tau}(u) \in \mathcal{U}_{\varepsilon / 2} \backslash \operatorname{int}\left(D_{\alpha} \cup \Psi^{c_{0}-\delta}\right)$ for some $\tau \in[0, T]$.

Case 2: $\left.\varphi^{t}(u) \in \Psi^{c_{0}+\delta} \backslash \operatorname{int}\left(\left(\mathcal{U}_{\varepsilon / 2} \cap H\right) \cup D_{\alpha} \cup \Psi^{c_{0}-\delta}\right)\right)$ for all $t \in[0, T]$.

In Case 1 we set $t_{2}:=\inf \left\{s>0: \varphi^{s}(u) \in \mathcal{U}_{\varepsilon / 2}\right\} \leqslant \tau$ and $t_{1}:=\sup \left\{s \in\left[0, t_{2}\right]: \varphi^{s}(u) \notin \operatorname{int}\left(\mathcal{U}_{\varepsilon} \cap H\right)\right\}$. Then

$$
\begin{aligned}
\left\|\varphi^{t_{2}}(u)-\varphi^{t_{1}}(u)\right\| & \geqslant\left|\varphi^{t_{2}}(u)-\varphi^{t_{1}}(u)\right|_{2} \\
& \geqslant \max \left\{\left|\left(\varphi^{t_{2}}(u)\right)^{+}-\left(\varphi^{t_{1}}(u)\right)^{+}\right|_{2},\left|\left(\varphi^{t_{2}}(u)\right)^{-}-\left(\varphi^{t_{1}}(u)\right)^{-}\right|_{2}\right\} \geqslant \frac{\varepsilon}{2} .
\end{aligned}
$$

Moreover, $\varphi^{t}(u) \in\left(\mathcal{U}_{\varepsilon} \backslash \mathcal{U}_{\varepsilon / 2}\right) \cap \Psi^{c_{0}+\delta}$ for $t_{1}<t<t_{2}$, and therefore (5.7) and the definition of $\varphi$ yield

$$
\begin{aligned}
\frac{\varepsilon}{2} & \leqslant\left\|\varphi^{t_{2}}(u)-\varphi^{t_{1}}(u)\right\| \leqslant \int_{t_{1}}^{t_{2}}\left\|\Psi^{\prime}\left(\varphi^{t}(u)\right)\right\| \mathrm{d} t \\
& \leqslant \frac{\varepsilon}{6 \delta} \int_{t_{1}}^{t_{2}}\left\|\Psi^{\prime}\left(\varphi^{t}(u)\right)\right\|^{2} \mathrm{~d} t=\frac{\varepsilon}{6 \delta} \Psi\left(\varphi^{t_{1}}(u)\right)-\Psi\left(\varphi^{t_{2}}(u)\right) .
\end{aligned}
$$

Hence $\Psi\left(\varphi^{t_{1}}(u)\right)-\Psi\left(\varphi^{t_{2}}(u)\right) \geqslant 3 \delta$ and therefore

$$
\Psi\left(\varphi^{\tau}(u)\right) \leqslant \Psi\left(\varphi^{t_{2}}(u)\right) \leqslant \Psi\left(\varphi^{t_{1}}(u)\right)-3 \delta \leqslant c_{0}-2 \delta .
$$

This yields a contradiction, since $\varphi^{\tau}(u) \notin \operatorname{int}\left(D_{\alpha} \cup \Psi^{c_{0}-\delta}\right)$ by assumption.

In Case 2 we find

$$
\Psi(u)-\Psi\left(\varphi^{T}(u)\right)=\int_{0}^{T}\left\|\Psi^{\prime}\left(\varphi^{t}(u)\right)\right\|^{2} \mathrm{~d} t \geqslant T \tau_{0}^{2}=3 \delta,
$$

by (5.6). Hence $\Psi\left(\varphi^{T}(u)\right) \in \Psi^{c_{0}-2 \delta}$, and we come to a contradiction again. Therefore (5.8) holds in any case, as claimed.

We now set

$$
\mathcal{E}:=\left\{u \in H \mid \varphi^{T}(u) \in D_{\alpha} \cup \Psi^{c_{0}-\delta}\right\} .
$$

Then $\mathcal{E} \subset H$ is closed, and it is strictly positively invariant under $\varphi$ as a consequence of Proposition 4.3(iii). Moreover, Lemma 5.2 yields

$$
\Psi^{c_{0}+\delta} \backslash \operatorname{int}\left(\mathcal{U}_{\varepsilon} \cap H\right) \subset \operatorname{int}(\mathcal{E}) .
$$

In order to find critical points of $\Psi$ in $H \backslash \mathcal{E}$ we need the following facts. Here we use the notation

$$
K_{c}^{*}=\left\{u \in H \backslash \mathcal{E}: \Psi(u)=c, \Psi^{\prime}(u)=0\right\} .
$$

Lemma 5.3. Consider $a, b \in \mathbb{R}$ with $0<a<b$ such that $K_{c}^{*}=\varnothing$ for $c \in(a, b)$ and $K_{a}^{*}$ consists of isolated points. Then $\Psi^{a} \cup \mathcal{E}$ is a strong deformation retract of $\left(\Psi^{b} \backslash K_{b}^{*}\right) \cup \mathcal{E}$.

Lemma 5.4. Suppose that $c>0$ and that $K_{c}^{*}$ consists of finitely many isolated critical points $u_{1}, \ldots, u_{m}$. Then there exists $\gamma>0$ such that

$$
H_{*}\left(\Psi^{c+\gamma} \cup \mathcal{E}, \Psi^{c-\gamma} \cup \mathcal{E}\right) \cong \bigoplus_{j=1}^{m} \mathcal{C}_{*}\left(u_{j}, \Psi\right) .
$$


Lemma 5.5. Consider $a, c \in \mathbb{R}$ with $a<c$, such that $K_{c}^{*}$ contains only finitely many isolated critical points $u_{1}, \ldots, u_{m}$ and $K_{b}^{*}=\varnothing$ for $b \in(a, c)$. Then there exists $\gamma>0$ such that

$$
H_{*}\left(\Psi^{c+\gamma} \cup \mathcal{E}, \Psi^{a} \cup \mathcal{E}\right) \cong \bigoplus_{j=1}^{m} \mathcal{C}_{*}\left(u_{j}, \Psi\right)
$$

Lemma 5.3 can be proved in a standard way along the lines of the proof of [13, Theorem 3.2], using the strict positive invariance of $\mathcal{E}$ under the flow $\varphi$. The proof of Lemma 5.4 proceeds analogously to the proof of [13, Theorem 4.2]. Lemma 5.5 just follows by combining Lemma 5.3 and Lemma 5.4.

For $\nu_{0}:=\Phi\left(w_{1}\right)+\Phi\left(w_{2}\right)<c_{0}+\frac{\delta}{2}$ we consider the continuous map

$$
\begin{aligned}
& g: S_{R} \times(0, \infty)^{2} \rightarrow \Psi^{\nu_{0}}, \\
& \left(y, s_{1}, s_{2}\right) \mapsto s_{1}\left(y * w_{1}\right)+s_{2} w_{2}
\end{aligned}
$$

which is well defined as a consequence of Proposition 3.3(iv). It follows from (3.11) and (3.12) that for $I_{b}:=[0, b]$ with $b>1+t_{0}, g$ induces a map of pairs

$$
g_{b}:\left(S_{R} \times I_{b}^{2}, S_{R} \times \partial I_{b}^{2}\right) \rightarrow\left(\Psi^{\nu_{0}} \cup \mathcal{E}, \mathcal{E}\right),
$$

and hence it induces a homomorphism

$$
g_{b_{*}}: \mathbb{Z} \cong H_{N+1}\left(S_{R} \times I_{b}^{2}, S_{R} \times \partial I_{b}^{2}\right) \rightarrow H_{N+1}\left(\Psi^{\nu_{0}} \cup \mathcal{E}, \mathcal{E}\right) .
$$

Let $i^{\nu}:\left(\Psi^{\nu_{0}} \cup \mathcal{E}, \mathcal{E}\right) \hookrightarrow\left(\Psi^{\nu} \cup \mathcal{E}, \mathcal{E}\right)$ be the inclusion, $v \geqslant v_{0}$. We first show

Lemma 5.6. If $b>0$ is sufficiently large, then for $v \geqslant 2 c_{+}+c_{-}+\frac{3}{4} \delta$ the map $\left(i^{v} \circ g_{b}\right)_{*}$ is trivial in dimension $N+1$.

Proof. We show that for $v \geqslant 2 c_{+}+c_{-}+\frac{3}{4} \delta$ and $b$ sufficiently large $i^{v} \circ g_{b}$ factorizes in the form

$$
i^{v} \circ g_{b}:\left(S_{R} \times I_{b}^{2}, S_{R} \times \partial I_{b}^{2}\right) \hookrightarrow\left(B_{R} \times I_{b}^{2}, B_{R} \times \partial I_{b}^{2}\right) \stackrel{g_{b}^{v}}{\longrightarrow}\left(\Psi^{\nu} \cup \mathcal{E}, \mathcal{E}\right),
$$

where $B_{R}:=B_{R}(0)$ and the first arrow denotes the inclusion. In order to define $g_{b}^{v}$, we fix $y_{0} \in S_{2 R}$. For $y \in B_{R}(0)$ and $s_{1}, s_{2} \in I_{b}$ we set

$$
g_{b}^{v}\left(y, s_{1}, s_{2}\right)= \begin{cases}s_{1}\left(\frac{|y|}{R}\left[\left(R \frac{y}{|y|}\right) * w_{1}\right]+\left(1-\frac{|y|}{R}\right)\left(y_{0} * w_{1}\right)\right)+s_{2} w_{2} & \text { if } y \neq 0 \\ s_{1} y_{0} * w_{1}+s_{2} w_{2} & \text { if } y=0 .\end{cases}
$$

By (3.11) this defines a continuous function $g_{b}^{v}: B_{R} \times I_{b}^{2} \rightarrow \Psi^{2 c_{+}+c_{-}+3 \delta / 4}$. If $b$ is chosen large enough, then $g_{b}^{v}\left(B_{R} \times \partial I_{b}^{2}\right) \subset D_{\alpha} \cup \Psi^{c_{0}-\delta} \subset \mathcal{E}$ by Lemma 3.1(ii). Thus, for $v \geqslant 2 c_{+}+c_{-}+\frac{3}{4} \delta$, we find that $g_{b}^{v}$ is a map of pairs

$$
g_{b}^{\nu}:\left(B_{R} \times I_{b}^{2}, B_{R} \times \partial I_{b}^{2}\right) \rightarrow\left(\Psi^{\nu} \cup \mathcal{E}, \mathcal{E}\right) .
$$

Note also that $i^{v} \circ g_{b}$ is just the restriction of $g_{b}^{v}$ to $S_{R} \times I_{b}^{2}$ so that (5.10) holds. We conclude by observing that $H_{j}\left(B_{R} \times I_{b}^{2}, B_{R} \times \partial I_{b}^{2}\right)=0$ for $j \geqslant 3$.

We now fix $b>1+t_{0}, I=I_{b}$ and $g=g_{b}$ according to Lemma 5.6. As an immediate consequence of Lemma 5.3 we obtain

Lemma 5.7. Let $v \in \mathbb{R}$ with $K_{v}^{*}=\varnothing$. 
(i) For $\gamma>0$ small enough there holds:

$H_{N+1}\left(\Psi^{\nu+\gamma} \cup \mathcal{E}, \mathcal{E}\right) \cong 0$ if and only if $H_{N+1}\left(\Psi^{\nu-\gamma} \cup \mathcal{E}, \mathcal{E}\right) \cong 0$.

(ii) If $v>c_{0}+\frac{\delta}{2}$, then for $0<\gamma<v-v_{0}$ small enough there holds:

$i_{*}^{\nu+\gamma} \circ g_{*}$ is trivial if and only if $i_{*}^{\nu-\gamma} \circ g_{*}$ is trivial.

Next we show

Proposition 5.8. For $v \in\left[v_{0}, c_{0}+\delta\right)$ the map

$$
\left(i^{\nu} \circ g\right)_{*}: H_{N+1}\left(S_{R} \times I^{2}, S_{R} \times \partial I^{2}\right) \rightarrow H_{N+1}\left(\Psi^{\nu} \cup \mathcal{E}, \mathcal{E}\right)
$$

is nontrivial.

Proof. Consider the sets $J=\left[1-t_{0}, 1+t_{0}\right] \subset I$ and $\mathcal{A}=I^{2} \backslash \operatorname{int}\left(J^{2}\right)$. By Lemma 3.1, (3.11) and (3.12) the map $i^{\nu} \circ g$ factorizes in the form

$$
\left(S_{R} \times I^{2}, S_{R} \times \partial I^{2}\right) \stackrel{i}{\longrightarrow}\left(S_{R} \times I^{2}, S_{R} \times \mathcal{A}\right) \stackrel{g^{v}}{\longrightarrow}\left(\Psi^{v} \cup \mathcal{E}, \mathcal{E}\right) .
$$

Here the inclusion $i$ induces an isomorphism

$$
i_{*}: H_{N+1}\left(S_{R} \times I^{2}, S_{R} \times \partial I^{2}\right) \rightarrow H_{N+1}\left(S_{R} \times I^{2}, S_{R} \times \mathcal{A}\right) .
$$

Now consider the map

$$
g_{1}:\left(S_{R} \times J^{2}, S_{R} \times \partial J^{2}\right) \hookrightarrow\left(S_{R} \times I^{2}, S_{R} \times \mathcal{A}\right) \stackrel{g^{v}}{\longrightarrow}\left(\Psi^{\nu} \cup \mathcal{E}, \mathcal{E}\right),
$$

where the first arrow is again the inclusion. By the preceding considerations it suffices to show that

$$
g_{1 *}: H_{N+1}\left(S_{R} \times J^{2}, S_{R} \times \partial J^{2}\right) \rightarrow H_{N+1}\left(\Psi^{\nu} \cup \mathcal{E}, \mathcal{E}\right) \quad \text { is nontrivial. }
$$

Note that by (3.10) the map $g_{1}$ factorizes in the form

$$
g_{1}:\left(S_{R} \times J^{2}, S_{R} \times \partial J^{2}\right) \stackrel{g_{2}}{\longrightarrow}\left(\left[\Psi^{\nu} \cup \mathcal{E}\right] \cap \mathcal{U}_{\varepsilon}, \mathcal{E} \cap \mathcal{U}_{\varepsilon}\right) \stackrel{j}{\longrightarrow}\left(\Psi^{\nu} \cup \mathcal{E}, \mathcal{E}\right),
$$

where $j$ again stands for the inclusion. Now since $\overline{\Psi^{\mathcal{V}} \backslash \mathcal{E}} \subset \operatorname{int}\left(\mathcal{U}_{\varepsilon} \cap H\right)$ by virtue of (5.9), the excision property yields that

$$
j_{*}: H_{N+1}\left(\left[\Psi^{v} \cup \mathcal{E}\right] \cap \mathcal{U}_{\mathcal{E}}, \mathcal{E} \cap \mathcal{U}_{\varepsilon}\right) \rightarrow H_{N+1}\left(\Psi^{v} \cup \mathcal{E}, \mathcal{E}\right)
$$

is an isomorphism. Hence it remains to show that

$$
g_{2 *}: H_{N+1}\left(S_{R} \times J^{2}, S_{R} \times \partial J^{2}\right) \rightarrow H_{N+1}\left(\left[\Psi^{\nu} \cup \mathcal{E}\right] \cap \mathcal{U}_{\varepsilon}, \mathcal{E} \cap \mathcal{U}_{\varepsilon}\right) \quad \text { is nontrivial. }
$$

For this we consider the continuous map

$$
\begin{aligned}
& h:\left(\Psi^{\nu} \cup \mathcal{E}\right) \cap \mathcal{U}_{\varepsilon} \rightarrow S_{R} \times[0, \infty)^{2} \\
& u \mapsto\left(R \frac{\beta\left(u^{+}\right)-\beta\left(u^{-}\right)}{\left|\beta\left(u^{+}\right)-\beta\left(u^{-}\right)\right|}, \tau_{0}\left(\varphi^{e_{T}(u)}(u)\right)\right),
\end{aligned}
$$

where $e_{T}: H \rightarrow[0, T]$ is defined by

$$
e_{T}(u):= \begin{cases}0, & u \in \mathcal{E} ; \\ \sup \left\{t \in[0, T] \mid \varphi^{t}(u) \notin \mathcal{E}\right\} & \text { elsewhere. }\end{cases}
$$

Note that $e_{T}$ is continuous since $\mathcal{E}$ is strictly positively invariant. By Proposition 3.3(i) the map $h$ is well defined. Moreover, if $\tau_{0}\left(\varphi^{e^{T}(u)}(u)\right)=(1,1)$, then $\varphi^{e_{T}(u)}(u) \in \mathcal{M}_{\Omega}$, hence $u \notin \mathcal{E}$ by Proposition 4.3(i). Thus $h$ is a map of pairs

$$
h:\left(\left[\Psi^{v} \cup \mathcal{E}\right] \cap \mathcal{U}_{\varepsilon}, \mathcal{E} \cap \mathcal{U}_{\varepsilon}\right) \rightarrow\left(S_{R} \times[0, \infty)^{2}, S_{R} \times\left([0, \infty)^{2} \backslash\{(1,1)\}\right)\right) .
$$


Moreover, since $g_{2}\left(S_{R} \times \partial J^{2}\right) \subset D_{\alpha} \cup \Psi^{c-\delta}$, the map

$$
k:=h \circ g_{2}:\left(S_{R} \times J^{2}, S_{R} \times \partial J^{2}\right) \rightarrow\left(S_{R} \times[0, \infty)^{2}, S_{R} \times\left([0, \infty)^{2} \backslash\{(1,1)\}\right)\right)
$$

satisfies $\left.k\right|_{S_{R} \times \partial J^{2}}=\mathrm{id}_{S_{R} \times \partial J^{2}}$. Hence, using the long exact sequences of the pairs $\left(S_{R} \times J^{2}, S_{R} \times \partial J^{2}\right)$ and $\left(S_{R} \times\right.$ $\left.[0, \infty)^{2}, S_{R} \times\left([0, \infty)^{2} \backslash\{(1,1)\}\right)\right)$ we conclude that

$$
k_{*}: H_{N+1}\left(S_{R} \times J^{2}, S_{R} \times \partial J^{2}\right) \rightarrow H_{N+1}\left(S_{R} \times[0, \infty)^{2}, S_{R} \times\left([0, \infty)^{2} \backslash\{(1,1)\}\right)\right) \cong \mathbb{Z}
$$

is an isomorphism. Thus $g_{2 *}$ is nontrivial, as required.

Finally we have:

Lemma 5.9. If $\mathcal{M}_{\Omega}^{c_{1}}$ consists of finitely many isolated critical points, then $H_{N+1}\left(\Psi^{c_{1}+\rho} \cup \mathcal{E}, \mathcal{E}\right) \cong 0$ for $\rho>0$ sufficiently small.

Proof. Suppose $\mathcal{M}_{\Omega}^{c_{1}}=\left\{u_{1}, \ldots, u_{m}\right\}$. Then by Lemma 5.5 and Proposition 4.1(ii) we have

$$
H_{N+1}\left(\Psi^{c_{1}+\rho} \cup \mathcal{E}, \mathcal{E}\right) \cong \bigoplus_{j=1}^{m} \mathcal{C}_{N+1}\left(u_{j}, \Psi\right) \cong 0
$$

for $\rho>0$ sufficiently small, since $N \geqslant 2$.

We may now complete the

Proof of Theorem 1.2. By Proposition 4.1 we may assume that $\mathcal{M}_{\Omega}^{c_{1}}$ contains at least one but only finitely many isolated critical points of $\Psi$, and these critical points have precisely two nodal domains.

We now define

$$
c_{2}:=\inf \left\{v>c_{1}: H_{N+1}\left(\Psi^{v} \cup \mathcal{E}, \mathcal{E}\right) \supsetneqq 0\right\}
$$

and

$$
c_{3}:=\sup \left\{v \geqslant c_{1}: i_{*}^{\nu} \circ g_{*} \text { is nontrivial }\right\}
$$

Then

$$
c_{1}<c_{2} \leqslant v_{0}<c_{0}+\delta \leqslant c_{3} \leqslant 2 c_{+}+c_{-}+\frac{3}{4} \delta
$$

by Lemma 5.6, Proposition 5.8 and Lemma 5.9. Moreover, Lemma 5.7 implies that $K_{c_{i}}^{*} \neq \varnothing$ for $i=2$, 3. Since $\delta<\min \left\{c_{+}, c_{-}\right\} \leqslant \bar{c}$, every critical point in $K_{c_{2}}^{*}$ has precisely two nodal domains by Proposition 4.1(iii). Moreover, since

$$
c_{3} \leqslant 2 c_{+}+c_{-}+\frac{3}{4} \delta<c_{+}+c_{-}+2 \min \left\{c_{+}, c_{-}\right\}
$$

by (5.1), a similar argument as in the proof of Proposition 4.1(iii) yields that every $u \in K_{c_{3}}^{*}$ has at most three nodal domains. Thus we obtain solutions $u_{2} \in K_{c_{2}}^{*}$ and $u_{3} \in K_{c_{3}}^{*}$ with the required properties.

\section{References}

[1] A. Ambrosetti, P.H. Rabinowitz, Dual variational methods in critical point theory and applications, J. Funct. Anal. 14 (1973) $349-381$.

[2] T. Bartsch, Critical point theory on partially ordered Hilbert spaces, J. Funct. Anal. 186 (2001) 117-152. 
[3] T. Bartsch, K.-C. Chang, Z.-Q. Wang, On the Morse indices of sign changing solutions of nonlinear elliptic problems, Math. Z. 233 (2000) 655-677.

[4] T. Bartsch, Z. Liu, T. Weth, Sign changing solutions to superlinear Schrödinger equations, Comm. Partial Differential Equations 29 (2004) 25-42.

[5] T. Bartsch, Z.-Q. Wang, On the existence of sign changing solutions for semilinear Dirichlet problems, Topol. Methods Nonlinear Anal. 7 (1996) 115-131.

[6] T. Bartsch, T. Weth, A note on additional properties of sign changing solutions to superlinear Schrödinger equations, Topol. Methods Nonlinear Anal. 22 (2003) 1-14.

[7] V. Benci, G. Cerami, Positive solutions of semilinear elliptic problems in exterior domains, Arch. Rational Mech. Anal. 99 (1987) $283-300$.

[8] V. Benci, G. Cerami, Multiple positive solutions of some elliptic problems via the Morse theory and the domain topology, Calc. Var. 2 (1994) 29-48.

[9] D. Cao, E.S. Noussair, Multi-peak solutions for a singularly perturbed semilinear elliptic problem, J. Differential Equations 166 (2000) 266-289.

[10] A. Castro, J. Cossio, J.M. Neuberger, A sign-changing solution for a superlinear Dirichlet problem, Rocky Mountain J. Math. 27 (1997) 1041-1053.

[11] A. Castro, J. Cossio, J.M. Neuberger, A minmax principle, index of the critical point, and existence of sign-changing solutions to elliptic BVPs, Electronic J. Differential Equations 2 (1998) 18.

[12] J. Chabrowski, Weak Convergence Methods for Semilinear Elliptic Equations, World Scientific, River Edge, NJ, 1999.

[13] K.C. Chang, Infinite Dimensional Morse Theory and Multiple Solution Problems, Progr. Nonlinear Differential Equations Appl., vol. 6, Birkhäuser, Boston, 1993.

[14] M. Conti, S. Terracini, G. Verzini, Nehari’s problem and competing species systems, Ann. Inst. H. Poincaré Anal. Non Linéaire 19 (2002) 871-888.

[15] V. Coti-Zelati, P.H. Rabinowitz, Homoclinic type solutions for a semilinear elliptic PDE on $\mathbb{R}^{N}$, Comm. Pure Appl. Math. XLV (1992) 1217-1269.

[16] K. Deimling, Ordinary differential equations in Banach spaces, in: Lecture Notes in Math., vol. 596, Springer, Berlin, 1978.

[17] B. Gidas, W. Ni, L. Nirenberg, Symmetry and related properties via the maximum principle, Comm. Math. Phys. 68 (1979) $209-243$.

[18] S.J. Li, Z.-Q. Wang, Mountain pass theorems in order intervals and multiple solutions for semilinear elliptic Dirichlet problems, J. Anal. Math. 81 (2000) 373-396.

[19] S.J. Li, Z.-Q. Wang, Lusternik-Schnirelman theory in partially ordered Hilbert spaces, Trans. Amer. Math. Soc. 354 (2002) $3207-3227$.

[20] Y. Li, L. Nirenberg, The Dirichlet problem for singularly perturbed elliptic equations, Comm. Pure Appl. Math. 51 (1998) 1445-1490.

[21] P. Lions, Symétrie et compacité dans les espaces de Sobolev, J. Funct. Anal. 49 (1982) 315-334.

[22] P. Lions, The concentration-compactness principle in the calculus of variations. The locally compact case. Part II, Ann. Inst. H. Poincaré Anal. Non Linéaire 1 (1984) 223-283.

[23] E. Müller-Pfeiffer, On the number of nodal domains for eigenfunctions of elliptic differential operators, J. London Math. Soc. (2) 31 (1985) 91-100.

[24] M. Struwe, Multiple solutions of differential equations without the Palais-Smale condition, Math. Ann. 261 (1982) $399-412$.

[25] M. Struwe, Variational Methods, Springer, Berlin, 1990.

[26] Z.-Q. Wang, On a superlinear elliptic equation, Ann. Inst. H. Poincaré Anal. Non Linéaire 8 (1991) 43-57. 